\title{
The structure and environment of young stellar clusters in spiral galaxies $\star, \star \star$
}

\begin{abstract}
S. S. Larsen ${ }^{\star \star \star}$
European Southern Observatory (ESO), Karl-Schwarzschild-Str. 2, 85748 Garching b. München, Germany

Received 17 October 2003 / Accepted 3 December 2003

Abstract. A search for stellar clusters has been carried out in 18 nearby spiral galaxies, using archive images from the Wide Field Planetary Camera 2 on board the Hubble Space Telescope. All of the galaxies have previously been imaged from the ground in UBVI. A catalogue of structural parameters, photometry and comments based on visual inspection of the clusters is compiled and used to investigate correlations between cluster structure, environment, age and mass. Least-squares fits to the data suggest correlations between both the full-width at half-maximum $(F W H M)$ and half-light radius $\left(R_{\text {eff }}\right)$ of the clusters and their masses $(M)$ at about the $3 \sigma$ level. Although both relations show a large scatter, the fits have substantially shallower slopes than for a constant-density relation ( $\operatorname{size} \propto M^{1 / 3}$ ). However, many of the youngest clusters have extended halos which make the $R_{\text {eff }}$ determinations uncertain. There is no evidence for galaxy-to-galaxy variations in the mean cluster sizes. In particular, the mean sizes do not appear to depend on the host galaxy star formation rate surface density. Many of the youngest objects (age $<10^{7}$ years) are located in strongly crowded regions, and about $1 / 3-1 / 2$ of them are double or multiple sources. The HST images are also used to check the nature of cluster candidates identified in a previous ground-based survey. The contamination rate in the ground-based sample is generally less than about $20 \%$, but some cluster identifications remain ambiguous because of crowding even with HST imaging, especially for the youngest objects.
\end{abstract}

Key words. galaxies: star clusters - galaxies: spiral - catalogs

\section{Introduction}

In previous papers (Larsen \& Richtler 1999, 2000; Larsen 1999; hereafter Papers I-III), we have studied populations of young stellar clusters in the disks of nearby spiral galaxies using ground-based imaging. That work was motivated by a desire to understand why some galaxies host young stellar clusters which are significantly more luminous (and, presumably, more massive) than open clusters in the Milky Way. Wellknown examples of galaxies with rich populations of luminous young clusters include a number of merger galaxies and starbursts (see e.g. compilation in Whitmore 2003), but there are also some relatively "normal" galaxies such as the Large Magellanic Cloud and M 33 which host a number of unusually (by Milky Way standards, at least) bright and massive young clusters (Shapley \& Nail 1951; Hodge 1961; Richtler 1993; Christian \& Schommer 1982, 1988; Chandar et al. 1999). In

\footnotetext{
* Full Tables 3-5, and 7 are only available in electronic form at the CDS via anonymous ftp to

cdsarc.u-strasbg.fr $(130.79 .128 .5)$ or via

http://cdsweb.u-strasbg.fr/cgi-bin/qcat? J/A+A/416/537

$\star \star$ Based on observations obtained with the NASA/ESA Hubble Space Telescope, obtained at the Space Telescope Science Institute, which is operated by the Association of Universities for Research in Astronomy, Inc., under NASA contract NAS 5-26555.

$\star \star \star$ e-mail: slarsen@eso.org
}

Paper III we concluded that the main driving factor behind these differences seems to be the star formation rate (SFR) of the host galaxy. Galaxies with high SFRs (per unit disk area) apparently form a larger fraction of their stars in massive, bound clusters. The presence of highly luminous clusters in galaxies with high SFRs may be - at least partially a size-of-sample effect, due to the rich cluster populations in such galaxies (Billett et al. 2002; Larsen 2002).

The study of stellar clusters is intimately linked to that of star formation in general. Observations show that a large fraction, if not the vast majority, of all stars are born in clusters (e.g. Carpenter 2000; Lada \& Lada 2003). This does not, however, imply that all embedded clusters are dynamically bound entities which survive emergence from their native molecular cloud cores and become observable at optical wavelengths. Lada \& Lada (2003) estimated that less than 4-7\% of local embedded clusters survive to become bound clusters of Pleiades age ( $\sim 10^{8}$ years), but this number may depend on environment. In Paper III we noted a steady increase with host galaxy areanormalised star formation rate $\left(\Sigma_{\mathrm{SFR}}\right)$ in the fraction of $U$-band light originating from clusters, ranging from well below $1 \%$ in galaxies with very low SFRs (like IC 1613) to several percent in starbursts. Meurer et al. (1995) found that on average about $20 \%$ of the UV light in a sample of starburst galaxies comes from young clusters. De Grijs et al. (2003) estimated that as much as $\sim 70 \%$ of the $B$-band light in the tidal tails 
of the "Tadpole" and "Mice" galaxies originates from young clusters or compact star-forming regions. In most "normal" star-forming galaxies, the young clusters contribute about $1 \%$ of the $U$-band light. Whether or not an embedded cluster remains gravitationally bound depends on the star formation efficiency within the proto-cluster cloud, as well as the timescale on which the gas is expelled (Elmegreen 1983; Kroupa 2001). It may therefore be more appropriate to view the fraction of optically visible young stars associated with clusters as a survival frequency, associated with the star formation efficiency, than a cluster formation efficiency per se (which is probably always close to $100 \%$ ).

While care must be taken when interpreting the above results, due to possible differences in the age distributions of the clusters and/or field stars, completeness limits, etc., it seems clear that the Solar neighbourhood samples only a small part of the conditions under which star formation takes place in the Universe. Fortunately, there are several star-forming galaxies available within a few Mpc, spanning a range in SFRs, morphological type etc., which can be studied in considerable detail with a combination of ground-based and space-based techniques. The original sample of 21 nearby spirals analysed in Papers I-III has since been augmented by an additional handful of galaxies observed with the 3-m Shane telescope at Lick Observatory (see Larsen 2002). However, on the ground-based images, clusters were only marginally resolved, and although significant efforts were made to weed out the most obvious contaminants, the cluster lists in Paper II should only be taken as provisionary. Many of the galaxies have now been imaged with the Wide Field Planetary Camera 2 (WFPC2) on board the Hubble Space Telescope (HST) for a variety of reasons, often with multiple pointings. The WFPC2 imaging not only provides a welcome check of the true nature of the sources identified as cluster candidates from the ground, but also allows the structure and immediate environment of individual clusters to be examined in much greater detail.

Several studies have indicated a remarkable uniformity in the sizes of stellar clusters over a wide range of masses, environments, ages and metallicities. The most robust measure of cluster size is the half-light or "effective" radius $\left(R_{\text {eff }}\right)$ which is expected to remain relatively stable over the lifetime of a cluster (Spitzer 1987). For Galactic globular clusters, $R_{\text {eff }}$ and luminosity are uncorrelated, although there is a trend of increasing cluster size with galactocentric distance $\left(R_{\mathrm{GC}}\right.$; van den Bergh et al. 1991). Using data from the compilation by Harris (1996), the median $R_{\text {eff }}$ is 3.0 pc. Similarly, the diameters and masses of Galactic open clusters show no strong correlation, with typical sizes only slightly smaller than those of globular clusters (Janes et al. 1988). For young clusters in the "Antennae" merger, Whitmore et al. (1999) found mean effective radii of $4 \pm 1$ pc. Zepf et al. (1999) estimated half-light radii of 5-10 pc for clusters in NGC 3256, perhaps slightly larger than for the Antennae, but again without any strong size-luminosity correlation. For globular clusters around early-type galaxies, typical effective radii are again 3-4 pc with no clear size-mass correlation (e.g. Kundu \& Whitmore 2001). The lack of a significant mass-size relation is puzzling, since one might a priori expect a cluster to form once the parent gas cloud reaches a certain density, independent of the total mass. If this initial gas density is reflected in the stellar density of the resulting cluster, one might naively expect the radius to scale with mass $(M)$ roughly as $R_{\mathrm{eff}} \propto M^{1 / 3}$. However, this is not what has generally been reported. From the above examples, it appears that star clusters typically have effective radii of a few $(\sim 3)$ pc, with a scatter of perhaps 1-2 pc. Exceptions are found, however, including the faint "Palomar"-type globular clusters in the outer part of the Galactic halo, and the "faint fuzzy" clusters recently discovered in a couple of nearby S0-type galaxies (Larsen \& Brodie 2000; Brodie \& Larsen 2002), which have larger effective radii ( $110 \mathrm{pc}$ ).

In this paper, the cluster candidates identified from the ground are first re-examined on archive WFPC2 images. Additional cluster candidates are then identified on the WFPC2 images and combined with ground-based photometry to produce a catalog of structural parameters and photometry for a sample of clusters. Relying on ground-based photometry limits the sample to relatively bright objects, but has the advantage of providing uniform photometric coverage of all clusters (even if crowding effects are more severe than in the HST data). In particular, most of the HST datasets do not include imaging in a $U$-band equivalent filter, which is essential for age-dating the clusters. However, the HST photometry may still be useful for some purposes and aperture photometry in an $r=0$. . 5 aperture is presented for the available bandpasses in a separate table for each cluster candidate. Each entry in the catalogue also contains various notes on the degree of crowding, close neighbours etc. based on a visual inspection of the candidates.

Because the HST images cover limited sections of the galaxies and span a huge range in exposure times and filters, the cluster sample presented here still cannot be considered complete in any sense. The completeness is a complicated function of crowding, cluster size, underlying surface brightness, exposure time in the HST images, bandpass, seeing in the groundbased data, galaxy distance, and probably many other factors which would be next to impossible to model in a satisfactory way. It should also be emphasized that what is presented is still a list of cluster candidates, which might contain contaminants (e.g. background galaxies). A definitive list of bona-fide clusters would require spectroscopic follow-up, but such an effort is beyond the scope of this paper and left for future studies. With this in mind, it is hoped that the catalogue may still provide a useful basis for further studies. As an example, it is used in Sect. 4 to investigate trends with age and mass in the cluster sizes, degree of crowding, and shape parameters.

\section{Data and reduction}

The search for HST archive data was concluded in October 2002 and only includes WFPC2 datasets which had been publicly released up until that time (Table 1). ACS data were available for a few galaxies, but have been excluded in order to allow a relatively simple and homogeneous reduction procedure.

Given the large volume of data, a fairly high degree of automatization had to be incorporated in the reduction procedures. When several exposures were available for a given field and filter, these were combined with the CRREJ task in the 
Table 1. Exposures. $\Delta(\alpha, \delta)$ indicates the offsets in right ascension and declination between the coordinate systems of the WFPC 2 frames and the ground-based data (tied to the USNO catalogue). The offsets are given in $\mathrm{s} / 15$ for right ascension and in arcseconds for declination (i.e. the $\cos \delta$ factor has not been applied to convert the offsets in $\alpha$ to true $\operatorname{arcsecs})$.

\begin{tabular}{|c|c|c|c|c|}
\hline Galaxy & $\begin{array}{l}\text { Prop. } \\
\text { Pros. }\end{array}$ & $\Delta(\alpha, \delta)$ & Filter & Exp. \\
\hline \multirow[t]{6}{*}{ NGC 628} & 9042 & $-0.06,-0.01$ & $F 450 W$ & $2 \times 230 \mathrm{~s}$ \\
\hline & & & $F 814 W$ & $2 \times 230 \mathrm{~s}$ \\
\hline & 8597 & $-0.0^{\prime} 05,+0.01$ & $F 606 \mathrm{~W}$ & $160+400 s$ \\
\hline & 5446 & $-0^{\prime} 02,+0^{\prime} 67$ & $F 606 \mathrm{~W}$ & $2 \times 80 \mathrm{~s}$ \\
\hline & 9676 & $-0.07,+00^{\prime} 37$ & $F 300 W$ & $2 \times 1000 \mathrm{~s}$ \\
\hline & & & $F 606 \mathrm{~W}$ & $3 \times 700 \mathrm{~s}$ \\
\hline \multirow[t]{2}{*}{ NGC 1156} & 9124 & $-0.04,-11^{\prime \prime} 05$ & $F 300 \mathrm{~W}$ & $2 \times 500 \mathrm{~s}$ \\
\hline & & & $F 814 W$ & $2 \times 40 \mathrm{~s}$ \\
\hline \multirow{9}{*}{ NGC 1313} & 8599 & $+00^{\prime} 05,-00^{\prime} 89$ & $F 814 W$ & $2 \times 300+50 \mathrm{~s}$ \\
\hline & 9042 & $+00^{\prime} 13,+00^{\prime} 20$ & $F 450 \mathrm{~W}$ & $2 \times 230 \mathrm{~s}$ \\
\hline & & & $F 606 \mathrm{~W}$ & $2 \times 230 \mathrm{~s}$ \\
\hline & 5446 & $+0.72,+9 !^{\prime} 43$ & $F 606 \mathrm{~W}$ & $2 \times 80 \mathrm{~s}$ \\
\hline & 8199 & - & $F 814 W$ & $2 \times 1300 \mathrm{~s}$ \\
\hline & 6341 & - & $F 439 \mathrm{~W}$ & $60 \mathrm{~s}$ \\
\hline & & & $F 555 W$ & $60 \mathrm{~s}$ \\
\hline & 6713 & - & $F 606 \mathrm{~W}$ & $2 \times 300 \mathrm{~s}$ \\
\hline & 6802 & - & $F 814 W$ & $700+800+499 s$ \\
\hline \multirow[t]{3}{*}{ NGC 2835} & 9042 & $-0.06,-0.43$ & $F 450 W$ & $2 \times 230 \mathrm{~s}$ \\
\hline & & & $F 814 W$ & $2 \times 230 \mathrm{~s}$ \\
\hline & 5446 & $-1{ }^{\prime \prime} 10,-0.50$ & $F 606 \mathrm{~W}$ & $2 \times 80 \mathrm{~s}$ \\
\hline \multirow[t]{3}{*}{ NGC 2997} & 5446 & $-0 ! 07,-4 !^{\prime \prime} 03$ & $F 606 \mathrm{~W}$ & $2 \times 80 \mathrm{~s}$ \\
\hline & 9042 & $-0 .^{\prime} 06,+0 !^{\prime} 19$ & $F 450 W$ & $2 \times 230 \mathrm{~s}$ \\
\hline & & & $F 814 W$ & $2 \times 230 \mathrm{~s}$ \\
\hline \multirow[t]{7}{*}{ NGC 3184} & 5446 & $-0 ! 71,-3 \prime^{\prime \prime} 02$ & $F 606 \mathrm{~W}$ & $2 \times 80 \mathrm{~s}$ \\
\hline & 8602 & $-0 ! 10,+0 ! 31$ & $F 555 W$ & $2 \times 350 \mathrm{~s}$ \\
\hline & 9041 & $-0.07,-1^{\prime \prime} 03$ & $F 555 W$ & $2 \times 80 \mathrm{~s}$ \\
\hline & & & $F 606 \mathrm{~W}$ & $4 \times 80 \mathrm{~s}$ \\
\hline & & & $F 675 W$ & $2 \times 80 \mathrm{~s}$ \\
\hline & & & $F 439 W$ & $2 \times 300 \mathrm{~s}$ \\
\hline & & & $F 814 W$ & $2 \times 300 \mathrm{~s}$ \\
\hline \multirow[t]{4}{*}{ NGC 3521} & 5446 & - & $F 606 \mathrm{~W}$ & $2 \times 80 \mathrm{~s}$ \\
\hline & 9042 & - & $F 450 W$ & $2 \times 230 \mathrm{~s}$ \\
\hline & & - & $F 814 W$ & $2 \times 230 s$ \\
\hline & 5972 & - & $F 555 \mathrm{~W}$ & $2 \times 1000 s$ \\
\hline \multirow[t]{7}{*}{ NGC 3621} & 5446 & $-1^{\prime \prime} 18,-5{ }^{\prime \prime} 49$ & $F 606 \mathrm{~W}$ & $2 \times 80 \mathrm{~s}$ \\
\hline & 5397 & $-0.06,+1{ }^{\prime \prime} 17$ & $F 555 W$ & $3 \times 900 \mathrm{~s}$ \\
\hline & & & $F 814 W$ & $4 \times 900 \mathrm{~s}$ \\
\hline & & & $F 439 W$ & $2 \times 900+180 \mathrm{~s}$ \\
\hline & & & $F 336 \mathrm{~W}$ & $2100+1500+180 \mathrm{~s}$ \\
\hline & 8584 & $+00^{\prime} 08,+1^{\prime \prime} 00$ & $F 555 W$ & $4 \times 1300 \mathrm{~s}$ \\
\hline & & & $F 814 W$ & $4 \times 1300 \mathrm{~s}$ \\
\hline \multirow[t]{9}{*}{ NGC 4258} & 8597 & $-0 ! 06,+0 ! 25$ & $F 606 \mathrm{~W}$ & $160+400 s$ \\
\hline & 8591 & $-0.04,-0 ! 49$ & $F 547 M$ & $4 \times 400 \mathrm{~s}$ \\
\hline & 6888 & $-0.07,+0 ! 28$ & $F 300 \mathrm{~W}$ & $2 \times 900+600 \mathrm{~s}$ \\
\hline & 5123 & $-0{ }^{\prime} 03,+00^{\prime} 14$ & $F 547 M$ & $1000+160 s$ \\
\hline & 9086 & - & $F 606 \mathrm{~W}$ & $3 \times 1300 \mathrm{~s}$ \\
\hline & & & $F 814 W$ & $3 \times 1300 \mathrm{~s}$ \\
\hline & 8805 & - & $F 606 \mathrm{~W}$ & $1000 \mathrm{~s}$ \\
\hline & 7277 & $+0.0^{\prime} 07,-0 .^{\prime} 34$ & $F 555 \mathrm{~W}$ & $6 \times 500 \mathrm{~s}$ \\
\hline & & & $F 814 W$ & $6 \times 500 \mathrm{~s}$ \\
\hline \multirow[t]{4}{*}{ NGC 5055} & 9042 & $-0 !^{\prime} 11,+00^{\prime} 15$ & $F 450 \mathrm{~W}$ & $2 \times 230 \mathrm{~s}$ \\
\hline & & & $F 814 W$ & $2 \times 230 \mathrm{~s}$ \\
\hline & 5446 & $+0 !^{\prime} 15,-1{ }^{\prime \prime} 52$ & $F 606 \mathrm{~W}$ & $2 \times 80 \mathrm{~s}$ \\
\hline & 8090 & - & $F 606 \mathrm{~W}$ & $500+700 \mathrm{~s}$ \\
\hline
\end{tabular}

STSDAS package in IRAF ${ }^{1}$. In most cases, no shifts in the image coordinate systems were required before combination but

${ }^{1}$ IRAF is distributed by the National Optical Astronomical Observatories, which are operated by the Association of Universities
Table 1. continued.

\begin{tabular}{|c|c|c|c|c|}
\hline Galaxy & Prop. & $\Delta(\alpha, \delta)$ & Filter & Exp. \\
\hline \multirow[t]{19}{*}{ NGC 5194} & 5652 & $+0 .^{\prime} 05,-0 .^{\prime} 37$ & $F 336 W$ & $3 \times 400 \mathrm{~s}$ \\
\hline & 5123 & $-0 .{ }^{\prime} 05,+0 .{ }^{\prime} 87$ & $F 547 M$ & $600+260 s$ \\
\hline & \multirow[t]{4}{*}{7375} & \multirow[t]{4}{*}{$+0 .^{\prime} 02,+0 .^{\prime} 05$} & $F 555 W$ & $2 \times 600 \mathrm{~s}$ \\
\hline & & & $F 814 W$ & $700+300 s$ \\
\hline & & & $F 336 W$ & $2 \times 600 \mathrm{~s}$ \\
\hline & & & $F 439 W$ & $600+500 s$ \\
\hline & \multirow[t]{3}{*}{5777} & \multirow[t]{3}{*}{$-0 . ' 13,-0 .{ }^{\prime} 54$} & $F 439 W$ & $2 \times 700 \mathrm{~s}$ \\
\hline & & & $F 555 W$ & $600 \mathrm{~s}$ \\
\hline & & & $F 814 W$ & $600 \mathrm{~s}$ \\
\hline & \multirow[t]{3}{*}{$9073 / 1$} & \multirow[t]{3}{*}{$-0.06,-0 .{ }^{\prime} 46$} & $F 450 W$ & $4 \times 500 \mathrm{~s}$ \\
\hline & & & $F 555 W$ & $4 \times 500 s$ \\
\hline & & & $F 814 W$ & $4 \times 500 s$ \\
\hline & \multirow[t]{3}{*}{$9073 / 2$} & \multirow[t]{3}{*}{$+0.00,+0 . .55$} & $F 450 W$ & $4 \times 500 \mathrm{~s}$ \\
\hline & & & $F 555 W$ & $4 \times 500 \mathrm{~s}$ \\
\hline & & & $F 814 W$ & $4 \times 500 \mathrm{~s}$ \\
\hline & 5419 & $+0 . .^{\prime} 01,-0 .{ }^{\prime} 30$ & $F 547 M$ & $230 \mathrm{~s}$ \\
\hline & \multirow[t]{2}{*}{9042} & \multirow[t]{2}{*}{$+0 .^{\prime} 12,-0 .^{\prime} 49$} & $F 606 \mathrm{~W}$ & $2 \times 230 s$ \\
\hline & & & $F 814 W$ & $2 \times 230 \mathrm{~s}$ \\
\hline & 7909 & - & $F 606 \mathrm{~W}$ & $2 \times 700 \mathrm{~s}$ \\
\hline \multirow[t]{2}{*}{ NGC 5204} & \multirow[t]{2}{*}{8601} & $+0 .^{\prime} 10,+0.23$ & $F 606 \mathrm{~W}$ & $600 \mathrm{~s}$ \\
\hline & & - & $F 814 W$ & $600 \mathrm{~s}$ \\
\hline \multirow[t]{6}{*}{ NGC 5236} & \multirow[t]{2}{*}{5971} & \multirow{2}{*}{00} & $F 606 W$ & $1100+1200 s$ \\
\hline & & & $F 814 W$ & $2 \times 1000+2 \times 1300 s$ \\
\hline & 7909 & $+0 .^{\prime} 07,-0 . .^{\prime} 02$ & $F 606 W$ & $2 \times 500 s$ \\
\hline & \multirow[t]{2}{*}{8234} & \multirow[t]{2}{*}{$+0 .^{\prime} 04,-0 .^{\prime} 18$} & $F 547 M$ & $180+350+400 s$ \\
\hline & & & $F 814 W$ & $160+200+350 s$ \\
\hline & 8805 & $+0 !^{\prime} 03,+0 .^{\prime} 48$ & $F 606 W$ & $2 \times 1000+2 \times 1400 s$ \\
\hline \multirow[t]{2}{*}{ NGC 5585} & 5446 & $+0.69,-11^{\prime \prime} 08$ & $F 606 \mathrm{~W}$ & $2 \times 80 s$ \\
\hline & 8599 & $+0 \prime^{\prime} 02,-0 .{ }^{\prime} 30$ & $F 814 W$ & $2 \times 300+40 s$ \\
\hline \multirow[t]{4}{*}{ NGC 6744} & \multirow[t]{2}{*}{9042} & $-0 .^{\prime} 17,+0 .^{\prime} 69$ & $F 450 \mathrm{~W}$ & $2 \times 230 \mathrm{~s}$ \\
\hline & & & $F 814 W$ & $2 \times 230 s$ \\
\hline & 8597 & $-0 !^{\prime} 12,+1^{\prime \prime} .21$ & $F 606 W$ & $160+400 s$ \\
\hline & 5446 & $+4^{\prime \prime} 84,-20 .^{\prime} 93$ & $F 606 \mathrm{~W}$ & $2 \times 80 s$ \\
\hline NGC 6946 & 6118 & $+0{ }^{\prime} 10,-0 \prime^{\prime} 05$ & $F 439 W$ & $2 \times 400 \mathrm{~s}$ \\
\hline & & & $F 555 W$ & $400 \mathrm{~s}$ \\
\hline & 8597 & $+0 .^{\prime} 09,-0 .^{\prime} 65$ & $F 606 W$ & $160+400 s$ \\
\hline & 8715 & $-0 . .^{\prime} 07,-0 .{ }^{\prime} 41$ & $F 439 W$ & $2 \times 1100 s$ \\
\hline & & & $F 555 W$ & $2 \times 300 s$ \\
\hline & & & $F 814 W$ & $2 \times 700 s$ \\
\hline NGC 7424 & 9042 & $-0 .{ }^{\prime} 09,+0 .{ }^{\prime} 29$ & $F 450 \mathrm{~W}$ & $2 \times 230 s$ \\
\hline & & & $F 814 W$ & $2 \times 230 s$ \\
\hline & 5446 & $-0 .{ }^{\prime} 67,-1^{\prime \prime} 47$ & $F 606 \mathrm{~W}$ & $2 \times 80 \mathrm{~s}$ \\
\hline & 8599 & $-0 .{ }^{\prime} 04,-1^{\prime \prime} 09$ & $F 814 W$ & $2 \times 300+40 s$ \\
\hline NGC 7793 & 9042 & $-0 .^{\prime} 10,-0 .^{\prime} 02$ & $F 450 W$ & $2 \times 230 s$ \\
\hline & & & $F 814 \mathrm{~W}$ & $2 \times 230 s$ \\
\hline & 8591 & $-0 .{ }^{\prime} 08,-0 .{ }^{\prime} 28$ & $F 547 M$ & $4 \times 400 \mathrm{~s}$ \\
\hline & 8599 & $-0 .^{\prime} 11,-0 .^{\prime} 73$ & $F 814 W$ & $2 \times 300+40 s$ \\
\hline & 5446 & $-0.23,-13^{\prime \prime} .25$ & $F 606 W$ & $2 \times 80 \mathrm{~s}$ \\
\hline & 8601 & - & $F 606 W$ & $600 \mathrm{~s}$ \\
\hline & & & $F 814 W$ & $600 \mathrm{~s}$ \\
\hline
\end{tabular}

when necessary, such shifts were applied using the IMSHIFT command in IRAF. For each WFPC2 pointing and each filter, objects were then detected with the DAOFIND task in DAOPHOT (Stetson 1987) running within IRAF. Objects with saturated pixels within a radius of 5 pixels were rejected. The detected sources were fitted with the ISHAPE profile-fitting algorithm (Paper II). ISHAPE models each source assuming an analytic model for the intrinsic profile of the source, convolved with the HST/WFPC2 point spread function (PSF). The $F W H M$ of the analytic model is iteratively adjusted until the

for Research in Astronomy, Inc. under contract with the National Science Foundation. 
best fit to the data is obtained. The initial round of profile fitting was carried out with a fitting radius of $r=5$ pixels and assuming a model of the form

$P(r) \propto\left(1+\left(r / r_{\mathrm{c}}\right)^{2}\right)^{-\alpha}$

with $\alpha=1.5$. Profiles of this type were shown by Elson et al. (1987) to provide good fits to young LMC clusters, and are hereafter referred to as "EFF" models (but note that the exponent $\alpha$ in Eq. (1) corresponds to $\gamma / 2$ in the definition by Elson et al. 1987). The WFPC2 PSF was modelled using version 5 of the TINYTIM package (Krist \& Hook 1997), including a convolution with the "diffusion kernel" to account for pixelto-pixel charge diffusion. PSFs were generated for each filter, and automatically selected by the reduction scripts to match the bandpasses used for the observations.

Because the main aim was to study the structure of spatially resolved objects, only objects which were detected at $>10 \sigma$ above the background noise were included in the analysis. Fainter objects would generally have too low $S / N$ for reliable size measurements, and would have increased the already substantial computing time required to fit the spatial profiles. Because of the vastly different exposure times, different bandpasses, background levels etc., the 10- $\sigma$ detection threshold does not translate into a well-defined completeness limit in terms of magnitude. A total of 82000 sources were detected and fitted, requiring a few days of CPU time on a $1.5 \mathrm{GHz}$ Pentium PC. At this stage, many objects appeared several times in the source list, being included in multiple HST pointings and/or bands.

After the initial round of profile fitting, the HST object lists were matched with the photometry data files from the ground-based surveys (all details concerning the reduction of the ground-based data are given in Paper II). Not only cluster candidates previously identified as such in the ground-based surveys, but all point-like sources in each ground-based CCD frame for which photometry was available, were matched. The matching was done by converting pixel coordinates measured on the WFPC2 images to celestial coordinates, using the METRIC task in the STSDAS package in IRAF. These coordinates were then matched with the coordinates of objects measured on the ground-based CCD images, tied to the US Naval Observatory meridian circle catalogue as provided by the ESO SkyCat Tool (Monet et al. 1998). In many cases, there were clear systematic offsets between the WFPC2 and USNO coordinate systems. These offsets were determined by displaying each individual WFPC2 frame and then marking the ground-selected cluster candidates contained within that frame. Because these would typically be among the brightest objects in the WFPC2 frames, and the offsets in general relatively minor $\left(\$ 1^{\prime \prime}\right)$, identifying the cluster candidates in the WFPC2 frames was usually unproblematic. The offsets between the WFPC2 and USNO coordinate systems are listed in Table 1 for each dataset. Note that large offsets were found for the exposures belonging to snapshot programme 5446. This is probably because these datasets were guided using the "Gyro Hold" mode, which provides less accurate pointing and tracking than the Fine Guidance Sensors on HST. A few WFPC2

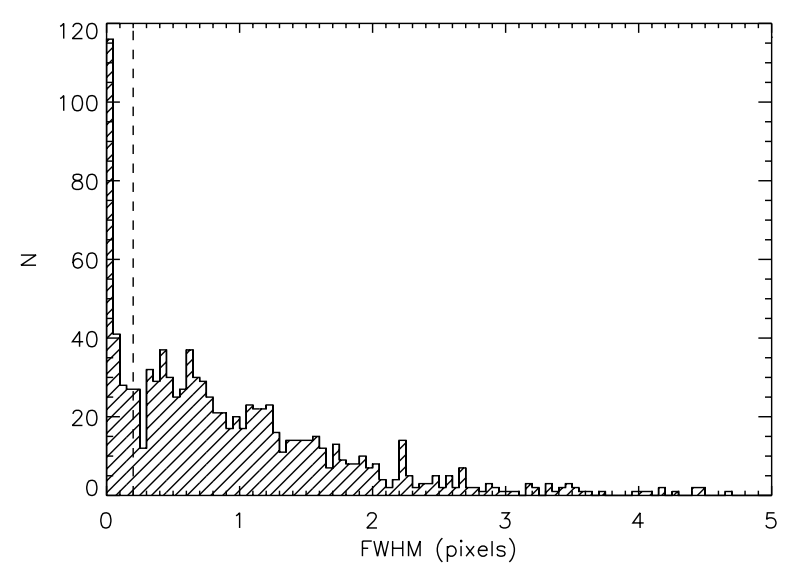

Fig. 1. Histogram of $F W H M$ values for objects fitted during the first round of profile fitting. The narrow sequence of unresolved objects with $F W H M \approx 0$ is clearly seen. The vertical dashed line indicates the criteria used to select cluster candidates.

pointings had no ground-based cluster candidates and were excluded from further analysis. For those pointings, no offsets are given in Table 1.

The initial round of profile fitting served to separate pointlike sources (likely stars) from extended ones (cluster candidates). Figure 1 shows the distribution of intrinsic FWHM values (in pixels). There is a very narrow peak of objects with $F W H M$ values close to 0 , although there is no sharp division between resolved and unresolved objects. For further analysis, a total of 3100 sources with $S / N>50$ (measured within the 5 pixels aperture on the WFPC2 images), $F W H M>0.2$ pixels (dashed line in Fig. 1) and ground-based photometry were selected. For comparison, the (undersampled) PSF of WFPC2 itself has a $F W H M$ of about 1.5 pixels. At a typical distance of $5 \mathrm{Mpc}$, the size cut corresponds to a $F W H M$ of $0.5 \mathrm{pc}$ or a core radius of about $0.25 \mathrm{pc}$. Many of these sources still represented multiple observations of the same object. Objects satisfying these criteria were again fitted, but this time allowing the power-law index $(\alpha)$ of the EFF profiles to vary as a free parameter. In order to better constrain $\alpha$, a fitting radius of 10 instead of 5 pixels was chosen for this second round. The choice of a 10 pixels fitting radius represents a compromise between reasonably accurate constraints on the fit parameters, reducing the effects of a non-uniform background, and computing time. Each source was fitted three times, using different initial guesses for $\alpha\left(\alpha_{0}=1.0,1.5\right.$ and 2.0). The values 1.0 and 1.5 roughly bracket those typically found for real star clusters (e.g. Elson et al. 1987 and Sect. 4.3), while an additional more extreme value of $\alpha_{0}=2$ was included in order to reduce any a priori bias in the measurements, in case some clusters have steeper slopes (although tests of the ISHAPE algorithm suggest that any dependence of the fitted parameters on the input guesses is relatively minor; see Sect. 2.2 below).

Finally, all cluster candidates fitted in the second round were visually inspected. In addition, all cluster candidates identified in the ground-based surveys and with HST imaging were inspected regardless of the $S / N$ in the HST images. The inspection was done by displaying a $4^{\prime \prime} \times 4^{\prime \prime}$ section of all images of each cluster candidate in an IDL widget, which also 
a (double/multiple):

n1313-540, n4258-1132, n5194-477

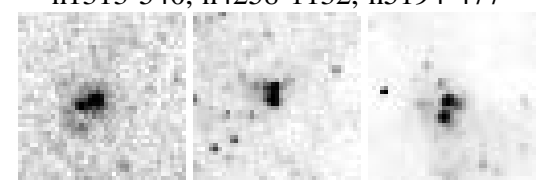

$\mathrm{b}$ (fainter neighbours):

n1313-304, n4258-1051, n5194-239

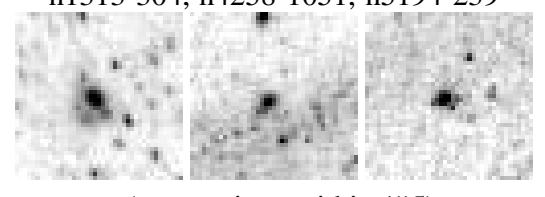

c (companions within 1".5):

n1313-471, n4258-454, n5194-121

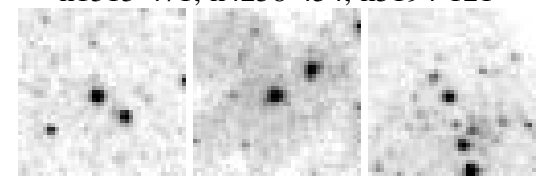

d (elongated):

n2835-625, n3184-277, n5194-351

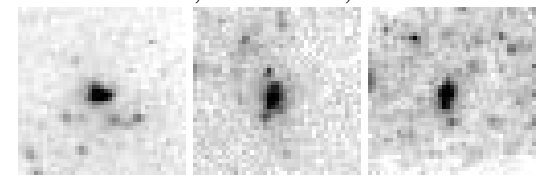

e (chain):

n5194-424, n5194-906, n628-1895

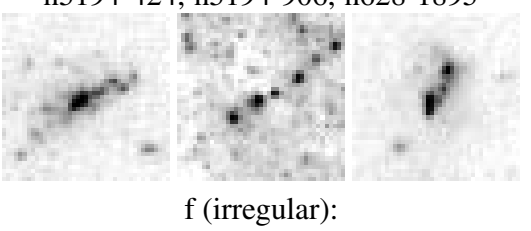

n1313-279, n2835-890, n7424-142

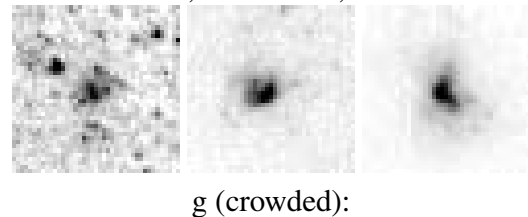

n5194-897, n1313-508, n2997-558

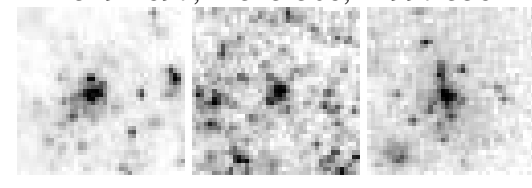

$\mathrm{h}$ (in group of more than 5 stars/clusters): n4258-776, n5194-433, n6946-1489

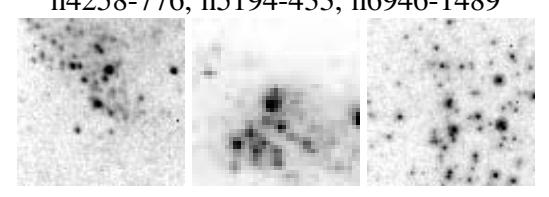

i (irregular background):

n4258-1060, n4258-794, n5194-394

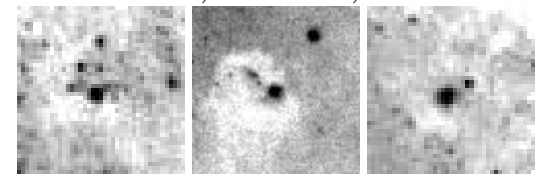

Fig. 2. Illustrations of comment codes a-i. Please see Table 2 for the full comments associated with each code. Each panel shows a 4 " $\times 4$ " section around the cluster candidate.
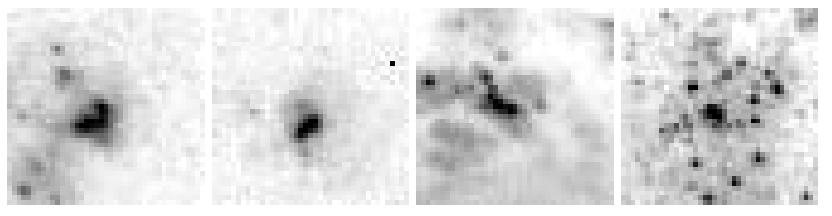

Fig. 3. Examples of objects classified as Type 2 (uncertain).

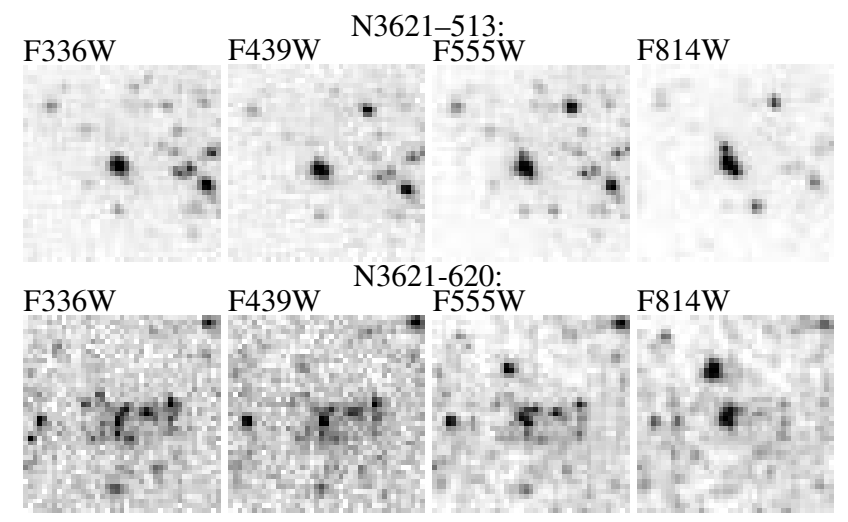

Fig. 4. Two examples of how the appearance of cluster candidates can change dramatically depending on bandpass.

Table 2. Explanation of comment codes.

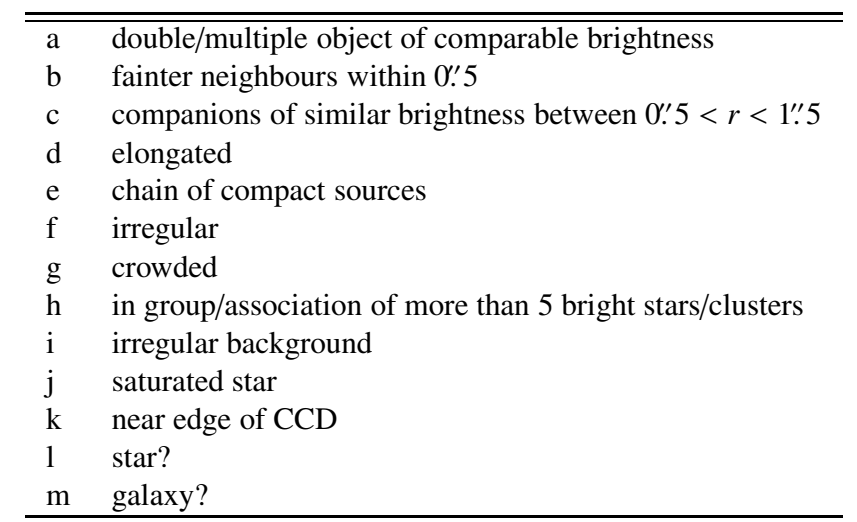

contained a number of check-boxes corresponding to the comment codes given in Table 2. Each object was also assigned to one of three types: 1) likely/certain cluster, 2) uncertain classification, 3) likely/certain non-cluster. A flag was set if the ISHAPE fit was unlikely to have resulted in meaningful structural parameters, as judged from the degree of crowding etc. The inspection process was repeated three times, each time displaying the cluster candidates in a different (random) order. At the end, the final object type and ISHAPE "Fit-OK" flag were determined as the most pessimistic of the three individual estimates. A comment code was included in the final list if selected in at least 2 out of the 3 inspection rounds.

Clearly, by its very nature any visual inspection involves an element of subjectivity. Very often, more than one comment applied to a given object. In such cases, all applicable comments were selected and included in the catalogue. Furthermore, resolution (and thus distance) effects may change the visual appearance of certain objects and thereby e.g. cause an object classified as "double" in a nearby galaxy to be labelled "elongated" 
Table 3. All clusters with $S / N>50$ on HST images. Photometry is from ground-based data while morphological data are from HST/WFPC2 images. The first 10 rows of the table are reproduced here; the full table (1358 rows) is only available in electronic form at the CDS.

\begin{tabular}{|c|c|c|c|c|c|c|c|c|}
\hline \multirow[t]{2}{*}{ ID } & RA (2000.0) & Dec (2000.0) & V & $U-B$ & $B-V$ & $V-I$ & $R_{\mathrm{GC}}$ & \\
\hline & $N$ & $F W H M(\mathrm{pc})$ & $\alpha$ & $R_{\mathrm{eff}}(\mathrm{pc})$ & $x / y$ & Code & Fit ok? & Comments \\
\hline \multirow[t]{2}{*}{ (1) } & (2) & (3) & (4) & (5) & (6) & (7) & (8) & \\
\hline & (9) & (10) & (11) & (12) & (13) & (14) & (15) & (16) \\
\hline \multirow[t]{2}{*}{ n1156-486 } & $2: 59: 41.29$ & $25: 14: 15.34$ & $16.97 \pm 0.01$ & $-0.96 \pm 0.01$ & $0.44 \pm 0.01$ & $-0.53 \pm 0.02$ & $0.30^{\prime}$ & \\
\hline & 1 & $8.55 \pm 0.81$ & $0.95 \pm 0.08$ & $12.42 \pm 1.24$ & $0.63 \pm 0.04$ & 1 & $\mathrm{~N}$ & $\mathrm{a}, \mathrm{f}$ \\
\hline \multirow[t]{2}{*}{ n1156-583 } & $2: 59: 42.85$ & $25: 14: 28.68$ & $17.41 \pm 0.01$ & $-0.29 \pm 0.01$ & $0.29 \pm 0.01$ & $0.65 \pm 0.01$ & $0.23^{\prime}$ & \\
\hline & 1 & $2.03 \pm 0.55$ & $0.88 \pm 0.05$ & $10.59 \pm 1.11$ & $0.97 \pm 0.03$ & 1 & $\mathrm{Y}$ & - \\
\hline \multirow[t]{2}{*}{ n1313-180 } & $3: 18: 15.12$ & $-66: 31: 55.29$ & $20.67 \pm 0.04$ & $0.11 \pm 0.15$ & $0.67 \pm 0.07$ & $0.98 \pm 0.06$ & $2.07^{\prime}$ & \\
\hline & 1 & $5.35 \pm 0.11$ & $0.89 \pm 0.01$ & $13.57 \pm 0.53$ & $0.95 \pm 0.03$ & 1 & $\mathrm{Y}$ & - \\
\hline \multirow[t]{2}{*}{ n1313-199 } & $3: 18: 17.49$ & $-66: 31: 36.73$ & $20.31 \pm 0.03$ & $0.03 \pm 0.08$ & $0.18 \pm 0.04$ & $0.49 \pm 0.05$ & $1.77^{\prime}$ & \\
\hline & 1 & $10.12 \pm 0.68$ & $2.09 \pm 0.58$ & $6.92 \pm 0.59$ & $0.78 \pm 0.03$ & 1 & $\mathrm{Y}$ & $\mathrm{d}$ \\
\hline \multirow[t]{2}{*}{ n1313-228 } & $3: 18: 18.46$ & $-66: 31: 22.76$ & $19.30 \pm 0.02$ & $0.23 \pm 0.06$ & $0.42 \pm 0.03$ & $0.57 \pm 0.02$ & $1.56^{\prime}$ & \\
\hline & 1 & $1.15 \pm 0.47$ & $5.64 \pm 5.24$ & $0.52 \pm 0.16$ & $0.53 \pm 0.39$ & 1 & Y & - \\
\hline \multirow[t]{2}{*}{ n1313-234 } & $3: 18: 07.67$ & $-66: 31: 19.06$ & $17.87 \pm 0.01$ & $-0.82 \pm 0.01$ & $0.34 \pm 0.01$ & $-0.78 \pm 0.02$ & $1.66^{\prime}$ & \\
\hline & 1 & $9.24 \pm 2.37$ & $0.87 \pm 0.60$ & $19.43 \pm 6.90$ & $0.92 \pm 0.02$ & 2 & $\mathrm{~N}$ & $a, f, g$ \\
\hline \multirow[t]{2}{*}{ n1313-239 } & $3: 18: 17.59$ & $-66: 31: 12.41$ & $19.86 \pm 0.04$ & $-0.07 \pm 0.08$ & $0.26 \pm 0.06$ & $0.38 \pm 0.08$ & $1.37^{\prime}$ & \\
\hline & 4 & $6.34 \pm 0.46$ & $1.29 \pm 0.32$ & $8.90 \pm 1.09$ & $0.90 \pm 0.02$ & 1 & $\mathrm{Y}$ & - \\
\hline \multirow[t]{2}{*}{ n1313-248 } & $3: 18: 16.05$ & $-66: 31: 06.91$ & $18.85 \pm 0.03$ & $1.21 \pm 0.24$ & $1.03 \pm 0.07$ & $1.11 \pm 0.04$ & $1.27^{\prime}$ & \\
\hline & 1 & $2.14 \pm 0.22$ & $70.80 \pm 90.78$ & $0.67 \pm 0.05$ & $0.22 \pm 0.03$ & 2 & $\mathrm{Y}$ & - \\
\hline \multirow[t]{2}{*}{ n1313-249 } & $3: 18: 12.45$ & $-66: 31: 06.38$ & $19.95 \pm 0.06$ & $0.10 \pm 0.10$ & $0.20 \pm 0.07$ & $0.60 \pm 0.08$ & $1.29^{\prime}$ & \\
\hline & 3 & $5.18 \pm 0.26$ & $1.25 \pm 0.08$ & $7.09 \pm 0.70$ & $0.92 \pm 0.03$ & 1 & $\mathrm{Y}$ & - \\
\hline \multirow[t]{2}{*}{ n1313-259 } & $3: 18: 15.55$ & $-66: 31: 02.25$ & $20.71 \pm 0.14$ & $-0.31 \pm 0.19$ & $0.26 \pm 0.19$ & $0.71 \pm 0.18$ & $1.19^{\prime}$ & \\
\hline & 2 & $1.40 \pm 0.42$ & $1.41 \pm 0.20$ & $1.44 \pm 0.05$ & $0.53 \pm 0.03$ & 1 & $\mathrm{Y}$ & - \\
\hline
\end{tabular}

in a slightly more distant one. In order to illustrate roughly what the individual comment codes in Table 2 represent, Fig. 2 shows $4^{\prime \prime} \times 4^{\prime \prime}$ sections around some clusters that are typical for each comment code. Figure 3 shows a few examples of objects classified as "uncertain". Another difficulty is that objects can appear quite different in different bandpasses. As an example, Fig. 4 shows two objects observed in $F 336 W$, $F 439 W, F 555 W$ and $F 814 W$. The $F 336 W$ and $F 814 W$ images of n3621-620 are hardly recognisable as the same object - the $F 336 W$ image just shows what might be a loose association of stars, while the $F 814 \mathrm{~W}$ image shows two fairly welldefined, compact sources. Conversely, n3621-513 looks compact and symmetric in $F 336 \mathrm{~W}$, while two nearby neighbours (perhaps red giants or supergiant stars) appear in $F 814 W$. If multi-wavelength data were available for all objects, one might adopt a consistent strategy for dealing with this problem, but in many cases data were only available in one band. It should therefore be borne in mind that the visual comment codes (and even the profile fits) depend on the bandpass in a way that is not easily predictable.

The final list of cluster candidates includes 1358 objects (Table 3). Columns (1)-(8) contain information from the ground-based data: coordinates, UBVI photometry and projected galactocentric distance (in arcmin). The photometry has been corrected for Galactic foreground extinction using the Schlegel et al. (1998) values and the extinction law in Cardelli et al. (1989). Note that the reddening corrections differ from those in Papers I-III, where Burstein \& Heiles (1984) values were used. In Papers I-III we used a relatively large aperture radius of 8 pixels (1'.5 and 3'. 2 for the NOT and Danish $1.54 \mathrm{~m}$ data, respectively) to avoid possible systematic effects in the integrated magnitudes due to the extendedness of the objects. However, it is now clear that most clusters are compact enough that this is not a major source of concern in ground-based imaging, and in the present paper I therefore use a smaller aperture radius of 4 pixels, the same as for the colours, for the groundbased magnitudes. Information derived from HST images is listed in Cols. (9)-(16): the number of individual detections of each cluster (where one "detection" is defined as the presence of the cluster in an image taken through a given filter under a given programme), $N$, is in Col. (9), followed by the fullwidth at half maximum $(F W H M)$ of the cluster profile derived from the ISHAPE fits, the exponent $\alpha$, the effective (half-light) radius $R_{\text {eff }}$, the minor/major axis ratio $(x / y)$, the object type (Col. 14), Fit-OK flag (Col. 15) and comments.

Unlike the classical King profiles (King 1962, 1966), the EFF models have no finite radius, and for $\alpha \leq 1$ the volume contained under the profile is infinite. For $\alpha$ only slightly larger than unity, the total volume converges very slowly, resulting in unrealistically large $R_{\text {eff }}$ values. To cope with these difficulties, the $R_{\text {eff }}$ values in Table 3 are computed for a finite outer radius of $50 \mathrm{pc}$, beyond which the luminosity profiles of young clusters become difficult to trace even in nearby galaxies such as the LMC (e.g. Elson et al. 1987). However, it is important to note that the estimates of $R_{\mathrm{eff}}$ are generally based on an extrapolation of the luminosity profiles beyond the fitting radius, and carry significant uncertainties especially when $\alpha \lesssim 1$. Instead of listing the $F W H M$, I could have given the core radius $r_{\mathrm{c}}$, since both are always defined. The reason for listing $F W H M$ is that there is some ambiguity in the definition of the core radius. Some authors define it as $F W H M / 2$, but it may also be defined e.g. as the scale radius $r_{\mathrm{c}}$ in Eq. (1). In order to avoid confusion, I will 
Table 4. Additional comments (only 5 sample entries given).

\begin{tabular}{ll}
\hline \hline ID & Comments \\
\hline $\mathrm{n} 1313-356$ & identification uncertain \\
$\mathrm{n} 1313-409$ & tracking error \\
$\mathrm{n} 1313-411$ & cluster members resolved? \\
$\mathrm{n} 2835-677$ & very elongated halo \\
$\mathrm{n} 2997-616$ & bad pixels \\
\hline
\end{tabular}

simply use the term $F W H M$ rather than "core radius" throughout the remainder of this paper. When more than one exposure was available for a cluster candidate, the shape parameters in Table 3 were obtained by weighting the measurements on each exposure by its $S / N$. As discussed above, the morphology of clusters can be quite wavelength-dependent, but cases where the determination of shape parameters is particularly uncertain can generally be recognized by the "Fit-OK" flag in Col. (15) of Table 3.

Errors were estimated as follows: for each exposure, the error on the shape parameters derived from that exposure were estimated as the standard deviation of the three individual fits. If only one exposure was available, this is the error listed in Table 3. When several exposures were available, the errors in Table 3 are the estimated standard errors on the mean of the weighted average. Some additional comments for a few objects, which did not fit into the codes in Table 2, are listed in Table 4.

\subsection{HST versus ground-based photometry}

HST photometry for each cluster candidate is given in Table 5. The HST magnitudes are given in the STMAG system, since transformation to the Johnson-Cousins system requires colour information which is not always available. The photometry was obtained with the PHOT task in DAOPHOT, using an $r=0.5$ aperture for the photometry and an $r=11^{\prime \prime} 0-1{ }^{\prime \prime} 5$ annulus for the background measurements. The 0.'5 aperture contains about $90 \%$ of the light from a point source (e.g. Holtzman et al. 1995), but because the objects measured here are extended, an even larger fraction of the total light will fall outside the 0.5 aperture. Thus, a total aperture correction of -0.2 mag has been applied to the magnitudes in Table 5. The exact correction will depend on the detailed spatial profiles of the clusters. In principle, this dependence might be estimated from the ISHAPE profile fits, but tying the photometry in Table 5 to the size measurements would make it difficult to backtrack these corrections. A constant -0.2 mag aperture correction is probably not far from the truth (Larsen 2002), and more sophisticated corrections can easily be applied if desired. In cases where several observations were available for a cluster in a given band, Table 5 lists the measurement with the smallest formal error.

In previous papers it has been documented that no systematic differences seem to be present between ground-based and HST-based colours for the cluster candidates (e.g. Larsen 2002), although a random scatter of 0.1-0.2 mag exists. For integrated magnitudes, on the other hand, an offset of a few $\times 0.1 \mathrm{mag}$ has been found between ground-based and HST magnitudes, in the sense that ground-based data tends to give brighter magnitudes. With the larger sample of clusters available here, this comparison can now be carried out in more detail. Of the clusters listed in Table 5, 1245 have data in at least one of the filters $F 547 M, F 555 W$ and $F 606 W$, all of which are reasonable approximations to the Johnson $V$-band. The mean difference $\left\langle\Delta V_{\text {Ground-WFPC2 }}\right\rangle$ between ground-based and WFPC2 photometry, including all objects with $F 547 M$, $F 555 W$ or $F 606 W$ HST data is -0.50 mag with a large scatter of $\sigma\left(\left\langle\Delta V_{\text {Ground-WFPC } 2}\right\rangle\right)=0.74 \mathrm{mag}$. This scatter is partly due to the fact that the ground-based photometry of some of the fainter clusters has large errors, but decreases only slightly (to $\sigma\left(\left\langle\Delta V_{\text {Ground-WFPC2 }}\right\rangle\right)=0.65 \mathrm{mag}$ ) if clusters with formal errors larger than 0.2 mag on the ground-based $V$ magnitudes are excluded. Thus, most of the errors are clearly of a systematic nature.

If the samples observed with the Danish $1.54 \mathrm{~m}$ telescope and the NOT are compared with the HST photometry separately, interesting differences emerge. The image quality of the data taken with the two telescopes differ significantly, with typical FWHM seeing values of 1'.5 and 0.'8, respectively (Larsen 1999). For the galaxies observed with the Danish $1.54 \mathrm{~m}$, the mean difference between ground-based and HST photometry is $\left\langle\Delta V_{\text {Ground-WFPC2 }}\right\rangle=-0.76 \pm 0.61 \mathrm{mag}$ (where the $0.61 \mathrm{mag}$ refer to the standard deviation around the mean, not the error on the mean value). For the NOT sample, the corresponding numbers are $\left\langle\Delta V_{\text {Ground-WFPC2 }}\right\rangle=-0.29 \pm 0.50 \mathrm{mag}$. Thus, while the scatter remains large even in the NOT data, the systematic difference relative to the HST photometry is clearly smaller than for the Danish $1.54 \mathrm{~m}$ data. A few individual, relatively isolated clusters observed with both the NOT and Danish $1.54 \mathrm{~m}$ telescope have been analysed in detail (Larsen et al. 2001; Larsen \& Richtler 2004, in preparation) and for these clusters there is good agreement between ground-based and HST magnitudes (within $\sim 0.1 \mathrm{mag}$ ). The differences between the mean magnitudes of the HST and ground-based samples are therefore not due to trivial zero-point errors in the photometric calibrations.

Clusters for which any of the comment flags in Table 2 are set might be expected to show poorer agreement with the ground-based data. Indeed, if such clusters are rejected then $\left\langle\Delta V_{\text {Ground-WFPC2 }}\right\rangle=-0.40 \pm 0.61 \mathrm{mag}$ for all clusters, and $\left\langle\Delta V_{\text {Ground-WFPC2 }}\right\rangle=-0.59 \pm 0.59 \mathrm{mag}$ and $\left\langle\Delta V_{\text {Ground-WFPC2 }}\right\rangle=-0.10 \pm 0.42 \mathrm{mag}$ for the Danish $1.54 \mathrm{~m}$ and NOT samples, respectively. Thus, the systematic difference between HST and ground-based photometry clearly decreases, albeit still with significant scatter. The remaining offsets can probably be attributed to contamination within the groundbased apertures which did not trigger any comment flags. In fact, if the HST photometry is instead carried out using an $r=1$.'0 aperture radius, assuming that such an aperture encircles all flux from the objects (i.e. applying no aperture corrections) then the mean offset with respect to the NOT data is only $\left\langle\Delta V_{\text {Ground-WFPC2 }}\right\rangle=-0.03 \pm 0.35 \mathrm{mag}$. Excluding clusters with comment codes, the difference decreases even further to $\left\langle\Delta V_{\text {Ground-WFPC2 }}\right\rangle=-0.003 \pm 0.33 \mathrm{mag}$.

A smaller subset of the clusters have observations in HST bandpasses that allow a comparison with the ground-based 
Table 5. HST photometry for the same clusters listed in Table 3. All magnitudes are in the STMAG system, measured in a $0{ }^{\prime} 5$ aperture and applying an aperture correction of $-0.2 \mathrm{mag}$. No corrections for foreground reddening have been applied. Only the first 10 rows are reproduced here.

\begin{tabular}{lcccccccc}
\hline \hline ID & $F 300 W$ & $F 336 W$ & $F 439 W$ & $F 450 W$ & $F 547 M$ & $F 555 W$ & $F 606 W$ & $F 814 W$ \\
\hline n1156-486 & $18.29 \pm 0.01$ & - & - & - & - & - & - & - \\
n1156-583 & $19.68 \pm 0.04$ & - & - & - & - & - & - & $18.90 \pm 0.01$ \\
n1313-180 & - & - & - & - & - & - & $21.11 \pm 0.01$ & - \\
n1313-199 & - & - & - & - & - & - & $21.05 \pm 0.01$ & - \\
n1313-228 & - & - & - & $19.51 \pm 0.01$ & - & - & $19.66 \pm 0.01$ & $20.18 \pm 0.01$ \\
n1313-234 & - & - & - & - & - & - & $19.28 \pm 0.01$ & - \\
n1313-239 & - & - & - & $20.38 \pm 0.02$ & - & - & $20.62 \pm 0.01$ & $21.01 \pm 0.01$ \\
n1313-248 & - & - & - & $19.32 \pm 0.01$ & - & - & $18.86 \pm 0.00$ & $18.96 \pm 0.00$ \\
n1313-249 & - & - & - & $20.28 \pm 0.02$ & - & - & $20.56 \pm 0.01$ & $20.98 \pm 0.01$ \\
$\mathrm{n} 1313-259$ & - & - & - & - & - & - & $21.09 \pm 0.02$ & $21.52 \pm 0.02$ \\
\hline
\end{tabular}

colours. For example, Holtzman et al. (1995) give transformations to Johnson $B-V$ colours for the $F 439 W$ and $F 555 W$ bandpasses, which are available for 190 of the clusters in Table 5. The mean offset between ground-based and HST $B-V$ colours is $\left\langle\Delta(B-V)_{\text {Ground-WFPC2 }}\right\rangle=-0.003$ mag with a scatter of $0.17 \mathrm{mag}$. For the Danish $1.54 \mathrm{~m}$ and NOT samples, the differences are $\left\langle\Delta(B-V)_{\text {Ground-WFPC2 }}\right\rangle=0.05 \pm 0.15 \mathrm{mag}$ and $\left\langle\Delta(B-V)_{\text {Ground-WFPC2 }}\right\rangle=-0.05 \pm 0.15 \mathrm{mag}$, respectively. This confirms that the ground-based colours are more accurate than magnitudes, presumably because the objects that contaminate the ground-based apertures tend to have similar ages and colours to the clusters themselves.

\subsection{Tests of the profile-fitting algorithm}

Tests of the ISHAPE code have been carried out in several previous papers, in particular in Paper II. The reader is referred to that paper and to the documentation included with the code for further details. However, because the ability to fit the EFF $\alpha$ parameter is a more recent addition, some tests of this particular feature are presented in the following.

First, artificially generated sources with known profiles were added to images of NGC 7793 and NGC 5194. The frames used for these tests were the WF4 chips from proposal 8591 (NGC 7793, F547M, $4 \times 400 \mathrm{~s}$ ) and proposal 7375 (NGC 5194, F555W, $2 \times 600 \mathrm{~s}$ ). The artificial sources were generated by convolving EFF profiles with the HST PSF, generated by TinyTim, and then added to the science images with the MKSYNTH program (Paper II). For each galaxy, artificial sources with $F W H M$ of 1 and 2 pc, $\alpha=1.0$ and 1.5, and magnitudes of $V=20,21$ and 22 were added. For each combination of these input parameters, 25 objects were added at random positions and then fitted with ISHAPE using three different values ( $\alpha_{0}=1.0,1.5$ and 2.0) for the initial guess of $\alpha$.

The test results are summarised in Table 6. For brevity, results are shown for $\alpha=1.0$ only, but the conclusions reached for $\alpha=1.5$ are essentially identical. The first four columns in each row give the input $V$ magnitude of the test objects, the $F W H M$ in pixels and pc, and the $\alpha$ parameter. The remaining columns list the mean $S / N$ of the test objects (within the fitting radius), followed by the fitted $F W H M$ and $\alpha$ values for the three initial guesses, $\alpha_{0}$, of the $\alpha$ parameter. The numbers in parentheses denote the rms deviation around the mean, excluding the two most deviating data points at each extreme. The last two columns list the mean standard deviation (" $\sigma_{3}$ ") of $F W H M$ and $\alpha$ for the three fits to a single object.

Neither the $F W H M$ nor the $\alpha$ values returned by ISHAPE show any systematic dependence on $\alpha_{0}$, and the mean fitted $F W H M$ and $\alpha$ are generally quite close to the input values. For $S / N \approx 50$, the rms scatter of the FWHM and $\alpha$ measurements are typically $0.15-0.20$ pixels and $0.10-0.15$ (dimensionless units), respectively. The mean standard deviation $\left(\sigma_{3}\right)$ of the three individual fits with different $\alpha_{0}$ is much smaller than the object-to-object rms scatter, indicating that the uncertainty due to a particular initial guess is smaller than the random measurement error. For typical cluster-like objects with $S / N \approx 50$, these tests suggest that the $F W H M$ and $\alpha$ parameter can be measured with an accuracy $\sim 20 \%$ and $10-15 \%$, respectively on a single image.

Note, however, that the error on the effective radius $R_{\mathrm{eff}}$ derived from the FWHM and $\alpha$ can be very much larger, especially if $\alpha \approx 1$. It is therefore important that any error selection be carried out on FWHM and $\alpha$, and not on the derived quantity $R_{\text {eff }}$.

As a final caution, the tests carried out here do not take into account any uncertainty on the PSF itself. In practice, this can be important even for HST images, since the so-called "diffusion" kernel makes an important contribution to the scattering of light between neighbouring pixels. While this effect is included in the modelling done by ISHAPE, the diffusion kernel has so far only been properly characterised for the $F 555 \mathrm{~W}$ filter. It is probably appropriate also for the $F 547 M$ and $F 606 \mathrm{~W}$ filters, but for bluer or redder passbands the true diffusion kernel could be significantly different.

\section{Contamination in the ground-based sample}

One of the motivations for this study was to test the reliability of the ground-based cluster identifications and quantify how much any mis-identifications might affect the specific luminosities for the cluster systems derived in Paper III. To this aim, Table 7 lists photometry and object classifications for all cluster candidates originally detected in the ground-based surveys, which are also included in the HST datasets. Of the 330 objects 
Table 6. Tests of the ISHAPE profile-fitting algorithm. For each combination of input parameters $(V=20 / 21 / 22, F W H M=1 \mathrm{pc} / 2 \mathrm{pc})$ the output fitted FWHM and $\alpha$ parameters are shown for three initial guesses of $\alpha\left(\alpha_{0}=1.0 / 1.5 / 2.0\right)$. Numbers in parentheses denote the object-to-object rms deviation around the mean values, excluding the two most deviating points at each extreme.

\begin{tabular}{|c|c|c|c|c|c|c|c|c|c|c|c|}
\hline \multirow{2}{*}{\multicolumn{4}{|c|}{ Input parameters }} & \multicolumn{8}{|c|}{ Output parameters } \\
\hline & & & & \multicolumn{2}{|c|}{$\alpha_{0}=1.0$} & \multicolumn{2}{|c|}{$\alpha_{0}=1.5$} & \multicolumn{2}{|c|}{$\alpha_{0}=2.0$} & \multicolumn{2}{|c|}{$\left\langle\sigma_{3}\right\rangle$} \\
\hline$V$ & $F W H M$, pix (pc) & $\alpha$ & $\langle S / N\rangle$ & $\langle F W H M\rangle$ & $\langle\alpha\rangle$ & $\langle F W H M\rangle$ & $\langle\alpha\rangle$ & $\langle F W H M\rangle$ & $\langle\alpha\rangle$ & $F W H M$ & $\alpha$ \\
\hline \multicolumn{12}{|c|}{ NGC 7793 (p8591, F547M) } \\
\hline 20.0 & $0.62(1.0)$ & 1.0 & 95.4 & $0.65(0.10)$ & $1.01(0.06)$ & $0.67(0.10)$ & $1.01(0.05)$ & $0.64(0.10)$ & $1.00(0.06)$ & 0.039 & 0.023 \\
\hline 20.0 & $1.25(2.0)$ & 1.0 & 96.7 & $1.27(0.14)$ & $0.99(0.08)$ & $1.29(0.15)$ & $1.00(0.08)$ & $1.27(0.13)$ & $0.99(0.07)$ & 0.033 & 0.019 \\
\hline 21.0 & $0.62(1.0)$ & 1.0 & 40.0 & $0.79(0.15)$ & $1.07(0.10)$ & $0.76(0.16)$ & $1.06(0.11)$ & $0.80(0.21)$ & $1.08(0.13)$ & 0.076 & 0.044 \\
\hline 21.0 & $1.25(2.0)$ & 1.0 & 41.1 & $1.25(0.25)$ & $1.01(0.13)$ & $1.25(0.27)$ & $1.00(0.13)$ & $1.22(0.31)$ & $0.99(0.15)$ & 0.086 & 0.044 \\
\hline 22.0 & $0.62(1.0)$ & 1.0 & 15.9 & $0.93(0.29)$ & $1.23(0.32)$ & $1.00(0.28)$ & $1.36(0.49)$ & $0.96(0.33)$ & $1.24(0.35)$ & 0.107 & 0.130 \\
\hline 22.0 & $1.25(2.0)$ & 1.0 & 17.1 & $1.35(0.48)$ & $1.15(0.46)$ & $1.41(0.44)$ & $1.17(0.35)$ & $1.49(0.45)$ & $1.17(0.41)$ & 0.148 & 0.139 \\
\hline \multicolumn{12}{|c|}{ NGC $5194(\mathrm{p} 7375, F 555 W)$} \\
\hline 20.0 & $0.25(1.0)$ & 1.0 & 133.6 & $0.33(0.06)$ & $1.03(0.05)$ & $0.31(0.05)$ & $1.03(0.05)$ & $0.31(0.06)$ & $1.02(0.05)$ & 0.037 & 0.024 \\
\hline 20.0 & $0.50(2.0)$ & 1.0 & 134.6 & $0.57(0.09)$ & $1.02(0.07)$ & $0.56(0.10)$ & $1.02(0.07)$ & $0.56(0.07)$ & $1.02(0.05)$ & 0.033 & 0.019 \\
\hline 21.0 & $0.25(1.0)$ & 1.0 & 57.4 & $0.41(0.17)$ & $1.11(0.18)$ & $0.37(0.12)$ & $1.08(0.14)$ & $0.39(0.12)$ & $1.07(0.13)$ & 0.054 & 0.039 \\
\hline 21.0 & $0.50(2.0)$ & 1.0 & 59.3 & $0.64(0.13)$ & $1.04(0.12)$ & $0.60(0.13)$ & $1.04(0.12)$ & $0.61(0.11)$ & $1.05(0.13)$ & 0.068 & 0.040 \\
\hline 22.0 & $0.25(1.0)$ & 1.0 & 26.0 & $0.54(0.19)$ & $1.21(0.36)$ & $0.57(0.24)$ & $1.38(0.82)$ & $0.58(0.20)$ & $1.39(0.78)$ & 0.080 & 0.112 \\
\hline 22.0 & $0.50(2.0)$ & 1.0 & 25.2 & $0.86(0.20)$ & $1.24(0.36)$ & $0.85(0.20)$ & $1.23(0.33)$ & $0.89(0.27)$ & $1.29(0.48)$ & 0.100 & 0.113 \\
\hline
\end{tabular}

Table 7. Clusters from ground-based survey reidentified in HST images. Photometry is from ground-based data. FWHM is the full-width-athalf-maximum in pc derived from $\operatorname{EFF} \alpha=1.5$ fits. The first 10 rows of the table are reproduced here; the full table (330 rows) is only available in electronic form at the CDS.

\begin{tabular}{|c|c|c|c|c|c|c|c|c|c|}
\hline ID & $\bar{V}$ & $\overline{U-B}$ & $B-V$ & $V-I$ & $N$ & $F W H M$ & Type & Fit ok? & Comments \\
\hline n1156-296 & $19.92 \pm 0.02$ & $-0.19 \pm 0.03$ & $0.12 \pm 0.03$ & $0.18 \pm 0.03$ & 1 & $0.20 \pm 0.00$ & 1 & $\mathrm{Y}$ & 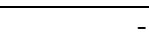 \\
\hline n1156-310 & $20.66 \pm 0.05$ & $-0.10 \pm 0.09$ & $0.18 \pm 0.07$ & $0.57 \pm 0.07$ & 2 & $6.58 \pm 2.38$ & 1 & $\mathrm{~N}$ & - \\
\hline n1156-331 & $19.85 \pm 0.04$ & $-0.67 \pm 0.05$ & $-0.03 \pm 0.05$ & $0.20 \pm 0.07$ & 1 & $0.08 \pm 0.00$ & 1 & Y & - \\
\hline n1156-348 & $18.43 \pm 0.01$ & $-0.79 \pm 0.02$ & $0.12 \pm 0.02$ & $0.61 \pm 0.02$ & 1 & $4.92 \pm 0.00$ & 2 & $\mathrm{~N}$ & $a, b$ \\
\hline n1156-356 & $20.27 \pm 0.02$ & $-0.06 \pm 0.04$ & $0.31 \pm 0.03$ & $0.55 \pm 0.02$ & 1 & $8.64 \pm 0.00$ & 2 & $\mathrm{~N}$ & $\mathrm{k}$ \\
\hline n1156-361 & $18.69 \pm 0.01$ & $-0.88 \pm 0.02$ & $-0.03 \pm 0.02$ & $0.45 \pm 0.02$ & 1 & $1.24 \pm 0.00$ & 1 & $\mathrm{Y}$ & - \\
\hline n1156-403 & $20.47 \pm 0.07$ & $-0.31 \pm 0.13$ & $0.24 \pm 0.11$ & $0.50 \pm 0.09$ & 2 & $7.20 \pm 4.74$ & 3 & $\mathrm{~N}$ & - \\
\hline n1156-433 & $20.45 \pm 0.07$ & $-0.24 \pm 0.11$ & $0.28 \pm 0.10$ & $0.68 \pm 0.09$ & 2 & $0.30 \pm 0.24$ & 2 & $\mathrm{~N}$ & $\mathrm{c}$ \\
\hline n1156-441 & $20.01 \pm 0.04$ & $0.27 \pm 0.10$ & $0.23 \pm 0.06$ & $0.42 \pm 0.07$ & 2 & $4.98 \pm 1.46$ & 1 & Y & - \\
\hline n1156-442 & $19.92 \pm 0.08$ & $-0.54 \pm 0.09$ & $-0.09 \pm 0.10$ & $0.17 \pm 0.12$ & 2 & $3.86 \pm 0.08$ & 1 & $\mathrm{Y}$ & - \\
\hline
\end{tabular}

listed in Table 7, 192 are also included in Table 3. The remaining 138 objects were excluded from the second round of fits (and Table 3) because of too low $S / N$ for the variable- $\alpha$ fits. However, size information is listed for all objects in Table 7 based on the first round of profile fits (with $\alpha=1.5$ ). Because only one fit was done for each exposure, meaningful error estimates are only available for cluster candidates with 2 or more detections. The mean and median error on the cluster FWHM values for objects with multiple measurements are $0.5 \mathrm{pc}$ and $0.26 \mathrm{pc}$, respectively, or $0.1-0.2$ pixels (for typical galaxy distances of $5 \mathrm{Mpc}$ ). This is consistent with the estimates of the accuracy of ISHAPE fits in Sect. 2.2 (see also Paper II).

The distribution of $F W H M$ values for objects listed in Table 7 is shown in Fig. 5. Only 6 objects were classified as "likely/certain non-clusters" during the visual inspection, but 69 objects (or about $21 \%$ of the sample) have $F W H M<0.5 \mathrm{pc}$ and are thus essentially unresolved even on the HST images. Unresolved objects are not necessarily individual, isolated stars, but can also be loose groupings of stars (OB associations etc.) where ISHAPE simply picks one star and fits it. This is the case for about 25 out of the 69 unresolved objects, or

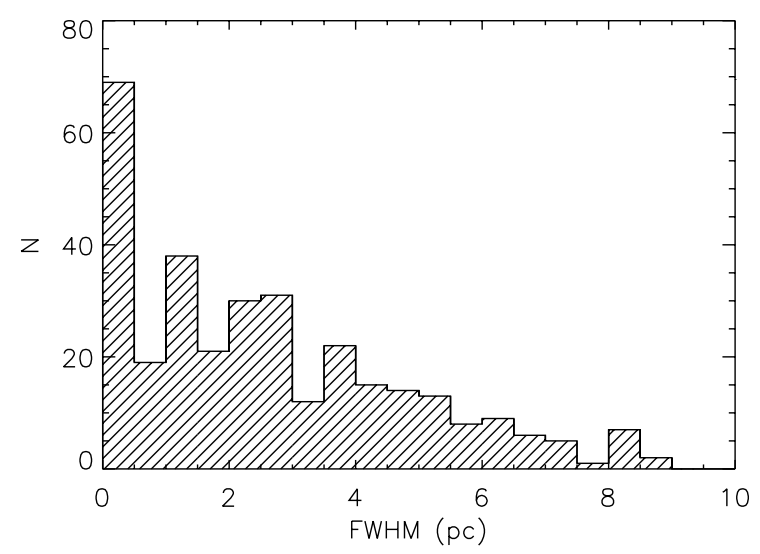

Fig. 5. Size distribution for cluster candidates identified in groundbased surveys.

about 1/3. A few examples are shown in Fig. 7. The size distribution in Fig. 5 does not appear strongly peaked at a particular value (except for the unresolved sources near $F W H M=0$ ), but spans a range from the resolution limit up to 8-10 pc. If objects with $F W H M<0.5$ pixels are excluded, the formal estimate of 


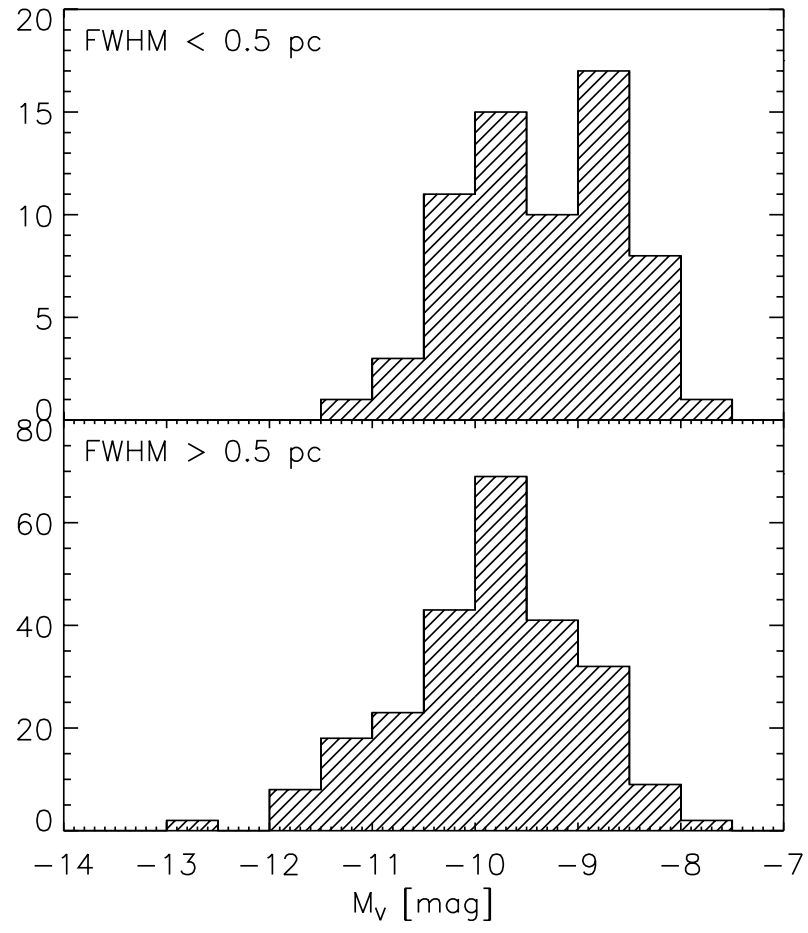

Fig. 6. Magnitude distributions for ground-selected cluster candidates with $F W H M<0.5 \mathrm{pc}$ (top) and $F W H M>0.5 \mathrm{pc}$ (bottom). Sizes are from EFF fits with fixed $\alpha=1.5$.

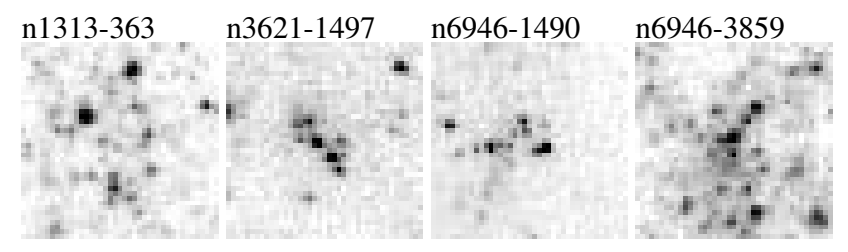

Fig. 7. Examples of objects identified as clusters from the ground, but which are unresolved according to the ISHAPE fits.

the mean FWHM is 3.8 pc. For an EFF model with $\alpha=1.5$, this corresponds to a half-light radius of $4.3 \mathrm{pc}$ (or $4.0 \mathrm{pc}$ if the profile is truncated at $50 \mathrm{pc}$ ), consistent with the typical sizes of young star clusters in the Milky Way and elsewhere. The mean $F W H M$ changes only slightly (to 3.9 pc) if objects with "Fit OK? = N" are excluded. The issue of cluster sizes will be discussed in more detail below for the full sample.

Figure 6 shows the distributions of absolute $M_{V}$ magnitudes for unresolved and resolved objects. While the magnitude distribution for resolved objects (bottom panel) does extend to brighter magnitudes than for unresolved ones, there are several unresolved objects brighter than $M_{V}=-10$. Such bright objects are unlikely to be individual stars, but most of them have the "c" comment set (companions within 1".5), indicating that the ground-based magnitudes are likely contaminated by nearby objects. Another possibility is that some of them are very compact star clusters. At magnitudes fainter than $M_{V}=-9$, Fig. 6 shows a clear excess of unresolved objects (26 out of 66 , or $39 \%$, compared to $21 \%$ for the whole sample), many of which may indeed be individual stars.

Another way of estimating the contamination fraction is to use the object types from the visual inspection. Of the

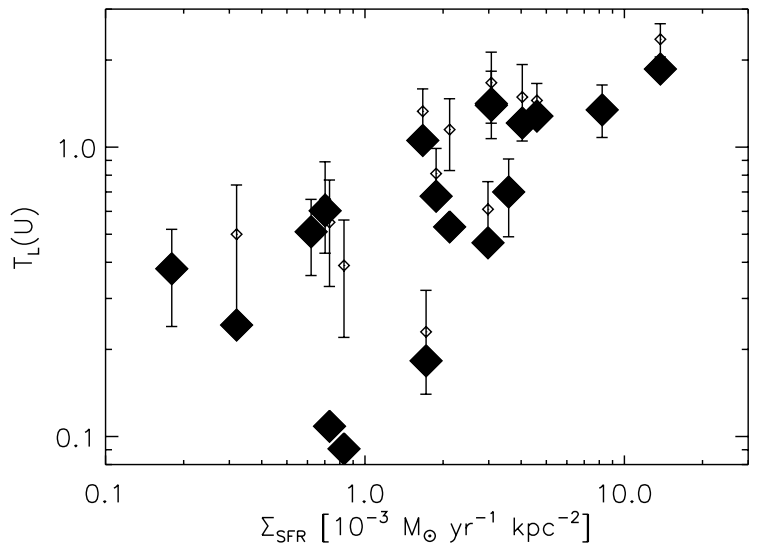

Fig. 8. Specific $U$-band luminosity $\left(T_{L}(U)\right)$ vs. area-normalised star formation rate $\left(\Sigma_{\mathrm{SFR}}\right)$ for original ground-based data and updated values (filled diamonds) based on contamination rates estimated from HST data (Table 8).

330 objects, 61 are labelled as type " 2 " (uncertain) or " 3 " (very likely non-cluster). Although classification as type "2" does not necessarily mean that the object in question is a non-cluster, these numbers again suggest a contamination rate of order $20 \%$ or less.

How does contamination of the cluster samples affect the relations between specific luminosity $\left(T_{L}(U)\right)$ and host galaxy properties derived in Paper III? If the contamination rate were the same in different galaxies, all specific luminosities would just be scaled down by a constant factor, leaving any relations involving $T_{L}(U)$ intact. However, it is possible that the ratio of clusters to potential contaminants varies from galaxy to galaxy. Table 8 lists the total number of cluster candidates identified from the ground $\left(N_{\text {tot }}\right)$ in each galaxy, followed by the number of ground-identified candidates contained within the WFPC2 images covering that galaxy $\left(N_{\mathrm{HST}}\right)$, and the number of objects among these that are unresolved $\left(N_{<0.5 \mathrm{pc}}\right)$. Based on the ground-based photometry, the $V$ - and $U$-band luminosities in resolved objects, relative to all objects in the HST images, are listed in the last two columns. With a couple of exceptions, exclusion of unresolved objects affects the luminosities of the cluster systems only weakly. The two most notable exceptions are NGC 2835 and NGC 5204. In Paper I, we noted that all 7 clusters in NGC 5204 are quite red and resulted in an unusually high specific frequency (number of clusters relative to the host galaxy luminosity). Now, it appears that most of these objects may in fact be foreground stars.

An updated version of the $T_{L}(U)$ vs. $\Sigma_{\text {SFR }}$ plot from Paper III is shown in Fig. 8. The original data are shown with error bars, while new updated $T_{L}(U)$ values, corrected according to Table 8 , are shown with filled diamonds. The basic trend for $T_{L}(U)$ to increase as a function of $\Sigma_{\mathrm{SFR}}$ is clearly preserved. The scatter increases somewhat after the exclusion of unresolved objects. However, with only a couple of clusters left in galaxies like NGC 2835 and NGC 5204 after exclusion of unresolved objects, $T_{L}(U)$ as defined in Paper III is probably no longer a good approximation to the true relative luminosity of the cluster system. To obtain a more useful number, one would 
Table 8. Contamination of ground-based sample for individual galaxies. $N_{\text {tot }}$ is the total number of cluster candidates identified in the ground-based surveys, $N_{\mathrm{HST}}$ is the subset of those candidates covered by HST images and $N_{<0.5 \mathrm{pc}}$ is the number of unresolved sources. The adopted distance moduli are also listed (see Paper I for references).

\begin{tabular}{lllllll}
\hline \hline Galaxy & $m-M$ & $N_{\text {tot }}$ & $N_{\mathrm{HST}}$ & $N_{<0.5 \mathrm{pc}}$ & \multicolumn{2}{c}{$L_{\mathrm{HST}} / L_{>0.5 \mathrm{pc}}$} \\
& & & & & $V$ & $U$ \\
\hline NGC 628 & 29.6 & 38 & 22 & 3 & 0.84 & 0.82 \\
NGC 1156 & 29.5 & 22 & 21 & 5 & 0.85 & 0.86 \\
NGC 1313 & 28.2 & 45 & 20 & 2 & 0.81 & 0.68 \\
NGC 2835 & 28.9 & 9 & 8 & 6 & 0.20 & 0.17 \\
NGC 2997 & 29.9 & 34 & 8 & 1 & 0.96 & 0.98 \\
NGC 3184 & 29.5 & 13 & 9 & 3 & 0.79 & 0.83 \\
NGC 3621 & 29.1 & 45 & 29 & 6 & 0.79 & 0.75 \\
NGC 4258 & 29.47 & 44 & 26 & 2 & 0.91 & 0.91 \\
NGC 5055 & 29.2 & 24 & 13 & 3 & 0.77 & 0.76 \\
NGC 5194 & 29.6 & 69 & 33 & 2 & 0.99 & 0.99 \\
NGC 5204 & 28.4 & 7 & 6 & 5 & 0.23 & 0.26 \\
NGC 5236 & 27.9 & 149 & 29 & 9 & 0.79 & 0.82 \\
NGC 5585 & 29.2 & 9 & 7 & 5 & 0.49 & 0.60 \\
NGC 6744 & 28.5 & 18 & 4 & 0 & 1.00 & 1.00 \\
NGC 6946 & 28.9 & 107 & 78 & 11 & 0.88 & 0.90 \\
NGC 7424 & 30.5 & 10 & 6 & 0 & 1.00 & 1.00 \\
NGC 7793 & 27.6 & 20 & 11 & 6 & 0.46 & 0.53 \\
\hline
\end{tabular}

likely have to probe to fainter magnitudes and thereby sample the cluster population more completely.

\section{The full sample}

\subsection{Cluster ages}

The broad-band colours of simple stellar populations (such as star clusters) are functions of both age and metallicity, with additional complications arising from unknown reddenings and stochastic effects due to the finite number of stars in a cluster (e.g. Girardi et al. 1995). However, for clusters younger than $\sim 10^{9}$ years it is still possible to obtain reasonably accurate photometric age estimates, especially if $U$-band data are included. For such clusters, metallicity effects are weak, except for a brief period around $10^{7}$ years when the integrated light is dominated by red supergiants. Here, cluster ages were obtained by fitting Bruzual \& Charlot (2000; priv. comm.) model colours to the observed $U B V I$ cluster colours. The SSP model fits were done by minimizing the rms deviation between model- and observed colours (weighted by their errors) as a function of age and reddening. In order to reduce the uncertainty on the age determinations, age estimates were made only for clusters with $\sigma_{B-V}<0.2 \mathrm{mag}, \sigma_{V-I}<0.2 \mathrm{mag}$ and $\sigma_{U-B}<0.3 \mathrm{mag}$.

The ages of individual clusters derived from broad-band colours should only be regarded as approximate. The groundbased apertures may be contaminated by objects other than the cluster candidate itself, and model uncertainties also make the absolute ages uncertain. However, the relative age ranking of clusters should still be reasonably reliable. Line emission can also affect the broad-band colours of very young objects,
Table 9. Mean FWHM and effective radii for clusters in galaxies.

\begin{tabular}{llccc}
\hline \hline Galaxy & $N$ & $\langle F W H M\rangle$ & $\left\langle R_{\text {eff }}\right\rangle(\alpha>1)$ & $\left\langle R_{\text {eff }}\right\rangle($ all $)$ \\
& & $\mathrm{pc}$ & $\mathrm{pc}$ & $\mathrm{pc}$ \\
\hline NGC 628 & 30 & $1.93 \pm 0.34$ & $3.65 \pm 0.55$ & $4.82 \pm 0.71$ \\
NGC 1313 & 67 & $3.48 \pm 0.25$ & $5.03 \pm 0.32$ & $5.83 \pm 0.44$ \\
NGC 2835 & 8 & $2.96 \pm 0.68$ & $5.18 \pm 1.22$ & $5.18 \pm 1.22$ \\
NGC 2997 & 11 & $3.89 \pm 0.75$ & $5.66 \pm 1.41$ & $8.73 \pm 1.75$ \\
NGC 3184 & 15 & $3.27 \pm 0.59$ & $3.44 \pm 0.60$ & $7.08 \pm 1.71$ \\
NGC 3621 & 49 & $2.61 \pm 0.18$ & $3.55 \pm 0.30$ & $4.37 \pm 0.48$ \\
NGC 4258 & 44 & $3.87 \pm 0.34$ & $4.71 \pm 0.47$ & $6.45 \pm 0.66$ \\
NGC 5194 & 126 & $3.12 \pm 0.15$ & $4.34 \pm 0.27$ & $5.48 \pm 0.36$ \\
NGC 5204 & 16 & $2.65 \pm 0.54$ & $3.31 \pm 0.73$ & $4.33 \pm 1.22$ \\
NGC 5236 & 80 & $2.04 \pm 0.15$ & $3.61 \pm 0.34$ & $4.84 \pm 0.47$ \\
NGC 5585 & 6 & $1.75 \pm 0.35$ & $3.79 \pm 0.16$ & $3.79 \pm 0.16$ \\
NGC 6744 & 10 & $2.52 \pm 0.59$ & $3.96 \pm 0.79$ & $3.96 \pm 0.79$ \\
NGC 6946 & 131 & $2.24 \pm 0.12$ & $3.22 \pm 0.22$ & $4.57 \pm 0.35$ \\
NGC 7424 & 12 & $5.24 \pm 1.25$ & $5.87 \pm 1.56$ & $5.87 \pm 1.56$ \\
NGC 7793 & 55 & $1.33 \pm 0.14$ & $2.26 \pm 0.47$ & $4.68 \pm 1.01$ \\
All & 661 & $2.68 \pm 0.07$ & $3.94 \pm 0.12$ & $5.24 \pm 0.17$ \\
\hline
\end{tabular}

and must be taken into account if accurate age estimates for objects younger than $\simeq 10^{7}$ years are required (e.g. Anders \& Fritze-v. Alvensleben 2003).

\subsection{Cluster sizes}

Table 9 lists the mean $F W H M$ and $R_{\text {eff }}$ for clusters in each galaxy. Only clusters classified as "Type 1" and with "Fit OK = YES" were included. The number of clusters in each galaxy satisfying these criteria is given in the second column. Because of the poorly defined $R_{\text {eff }}$ for clusters with $\alpha<1$, such clusters were excluded before computing the average $R_{\text {eff }}$ values in Table 9. Objects with very steep envelope slopes $(\alpha>5)$ represent clear outliers (see Sect. 4.3 below) and were also excluded from further analysis. The mean values given in the table are unweighted. This is because there is a correlation between the cluster sizes and their associated errors (the relative errors remain roughly constant), which would lead to a strong bias in the mean if the size measurements were error-weighted.

The mean effective radius is $\left\langle R_{\text {eff }}\right\rangle=3.94 \pm 0.12 \mathrm{pc}$, perhaps slightly larger compared to those for the ground-selected sample (Sect. 3) and other young and old star clusters. This may be due to the fact that the clusters are assumed to follow a single power-law out to a total radius of $50 \mathrm{pc}$, while in reality the behaviour at large radii is poorly constrained. If the cluster profiles decline more rapidly at large radii the effective radii would decrease, especially for objects with $\alpha$-values close to 1. Also, the size cut imposed in order to exclude point sources may introduce a bias against the most compact clusters. In the last column of Table 9, the $\alpha>1$ requirement is abandoned. Clearly, this leads to an increase in the mean effective radii, but it is stressed that the $R_{\text {eff }}$ values for these objects are very uncertain and depend strongly on the adopted outer radii.

The mean $F W H M$ and $R_{\text {eff }}$ from Table 9 are plotted versus parent galaxy distance modulus in Fig. 9. The sizes do 


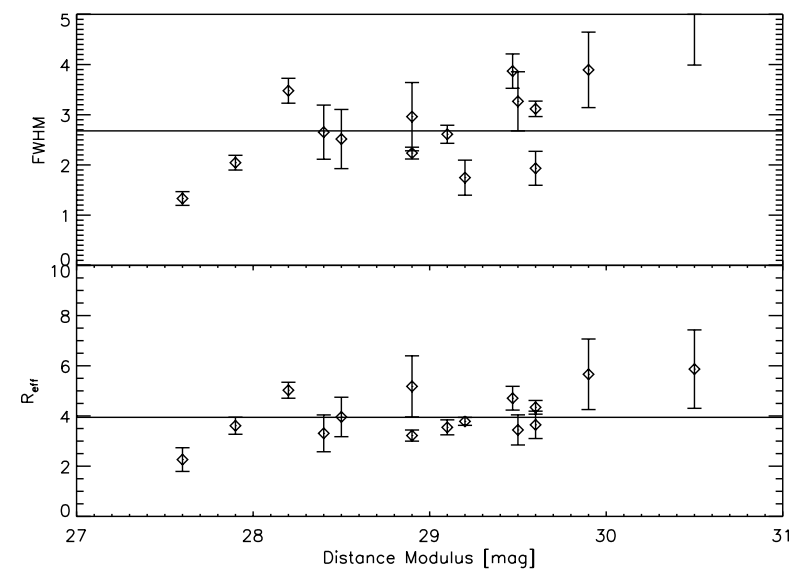

Fig. 9. Mean FWHM (top) and effective radii (bottom) for stellar clusters versus parent galaxy distance modulus.

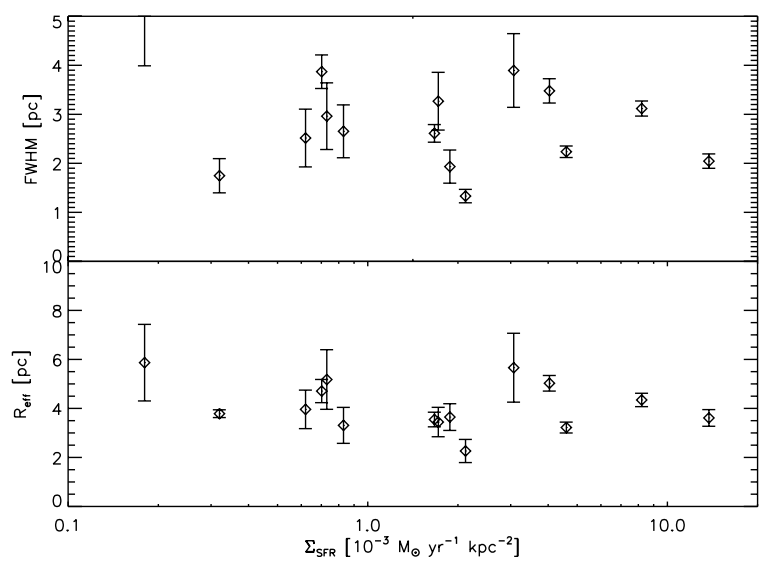

Fig. 10. Mean $F W H M$ (top) and effective radii (bottom) for stellar clusters versus parent galaxy $\Sigma_{\mathrm{SFR}}$ (derived from IRAS far-infrared luminosities, as described in Paper III).

show some correlation with parent galaxy distance, possibly due to a less than perfect correction for the PSF. It is also possible that a larger fraction of the objects detected in more nearby galaxies are individual stars, rather than clusters, which made it into the list of cluster candidates despite the size cut. Contributing to this effect, the number of individual stars bright enough to be detected in the ground-based photometry would increase at smaller distances. Furthermore, the fixed size cut at $F W H M=0.2$ pixels corresponds to a different physical cluster size in different galaxies, ranging from $0.3 \mathrm{pc}$ (core radius $\sim 0.15 \mathrm{pc}$ ) in NGC 7793 to $1.2 \mathrm{pc}$ (core radius $\sim 0.6 \mathrm{pc}$ ) in NGC 7424.

Figure 10 shows the mean cluster sizes as a function of the area-normalised host galaxy star formation rate, $\Sigma_{\mathrm{SFR}}$ (see Paper III). The scatter in the $F W H M$ plot is somewhat larger than for the $R_{\text {eff }}$ values, but neither $F W H M$ nor $R_{\text {eff }}$ shows any obvious correlation with $\Sigma_{\mathrm{SFR}}$. Below, the data for all galaxies is combined and analysed collectively in order to improve statistics, but in order to reduce possible systematic effects due to different distances, clusters in the closest (NGC 7793) and two most distant galaxies (NGC 2997 and NGC 7424) are excluded for the analysis of structural parameters. Thus, in
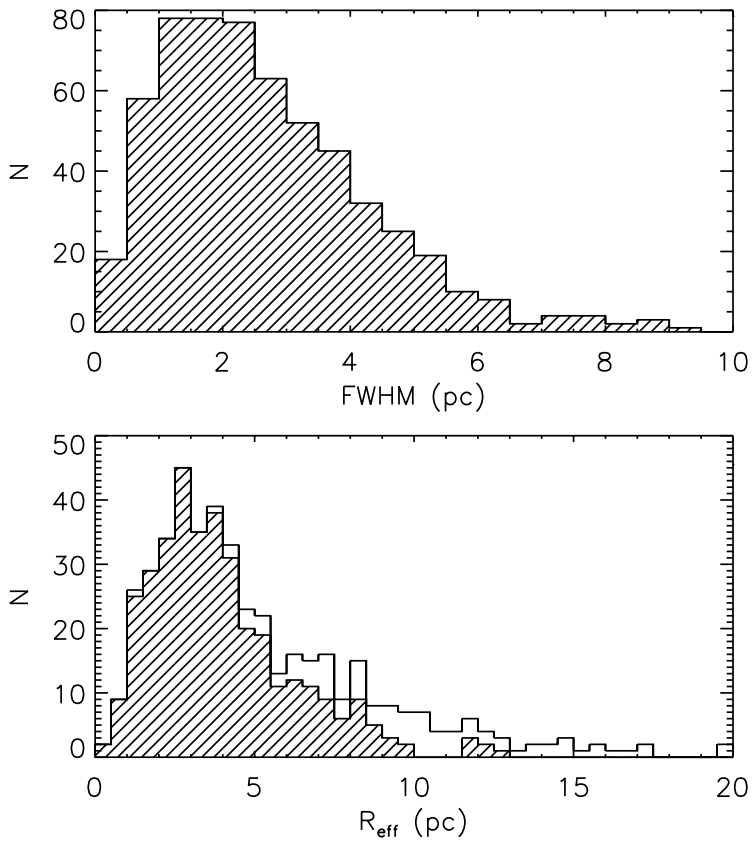

Fig. 11. Distributions of $F W H M$ and effective radii for the combined cluster samples. In the lower panel, the hatched and outlined histograms are for clusters with $1<\alpha<5$ and $\alpha<5$, respectively.

summary the selection parameters applied to the cluster candidates in Table 3 for the following analysis are:

- Object type $=1$ (certain/very likely cluster);

- Fit = OK;

- When $R_{\text {eff }}$ is involved: require $1<\alpha<5$;

- Photometric errors: $\sigma_{B-V}<0.2 \mathrm{mag}, \sigma_{V-I}<0.2 \mathrm{mag}$ and $\sigma_{U-B}<0.3 \mathrm{mag}$;

- Exclude NGC 2997, NGC 7424 and NGC 7793.

Figure 11 shows the distributions of $F W H M$ and $R_{\text {eff }}$ values for the combined sample. The paucity of objects with $F W H M<1 \mathrm{pc}$ is partly a selection effect, due to the size cut at $F W H M=0.2$ pixels, so it is not clear that the distribution of $F W H M$ even has a well-defined peak. However, most clusters have $F W H M<6-8 \mathrm{pc}$, similar to the range spanned by the ground-selected clusters (Fig. 5). The $R_{\text {eff }}$ distribution is more complicated to interpret because the cut in FWHM does not translate to a unique $R_{\text {eff }}$ but depends on $\alpha$. For a typical $\alpha \sim 1.3$ (see below), a $F W H M$ of $1 \mathrm{pc}$ corresponds to $R_{\mathrm{eff}}=1.6 \mathrm{pc}$, but this increases to $R_{\text {eff }}=3 \mathrm{pc}$ for $\alpha=1.1$ and $R_{\mathrm{eff}}=8 \mathrm{pc}$ for $\alpha=0.9$. However, there is a fairly rapid drop-off in the distribution for $R_{\text {eff }}>5 \mathrm{pc}$, and the vast majority of the clusters have $R_{\text {eff }}<10 \mathrm{pc}$. The mean $F W H M$ and $R_{\text {eff }}$ values given in Table 9 and shown in Figs. 9 and 10 should clearly be interpreted with caution.

\subsection{Envelope slope versus age}

Figure 12 shows the distribution of $\alpha$ values for clusters in four different age bins. For reference, typical errors of 0.15 on $\alpha$ are indicated (cf. Sect. 2.2). The distribution of $\alpha$ values is peaked around 1.2-1.3 in all four bins, but there may be a trend of mean $\alpha$ increasing with age, at least in the sense that the youngest age 


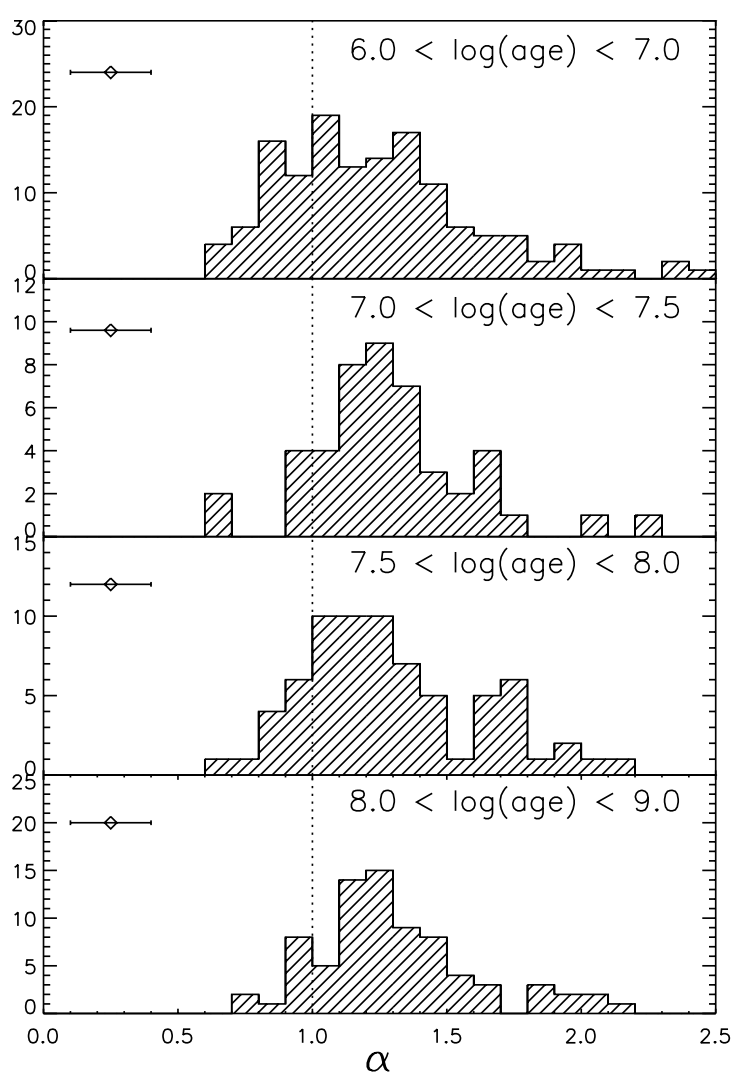

Fig. 12. Distribution of envelope slopes in four age bins.

bin appears to contain a higher fraction of clusters with $\alpha<1$. One important caveat in this comparison is that the mean mass is likely to increase from the youngest to the older age bins, due to the increase in $M / L$ ratio with age. Thus, in principle the difference between the $\alpha$-distributions in Fig. 12 might be due to the different mass ranges sampled in each bin, rather than being an evolutionary effect. To test whether this might be the case, Fig. 13 displays the $\alpha$-distributions in two age intervals, but now also divided into different mass intervals. The number of clusters in each panel is quite small, but the excess of clusters with $\alpha<1$ seems to be present for $\log ($ age $)<7.0$ in all three mass bins. This suggests that the difference between the $\alpha$-distributions in Fig. 12 is not just an effect of the different mass intervals covered at different ages.

Other authors have previously noted that very young clusters tend to be surrounded by relatively extended envelopes with more light at large radii than a King profile. Elson et al. (1987) estimated that as much as $50 \%$ of the mass in young LMC clusters may reside in unbound halos. In the Milky Way, a large fraction of the youngest open clusters also have very large radii and may be unbound and in the process of dispersing away (Janes et al. 1988). Whitmore et al. (1999) showed radial profiles for three clusters in the Antennae, illustrating a gradual transition from extended envelopes with no well-defined outer radius (for the highly luminous "Knot S", only a few Myr old) to older clusters where a tidal cut-off becomes apparent. A similar extended halo was observed for a very luminous, $15 \mathrm{Myr}$ old cluster in NGC 6946 (Larsen et al. 2001). The structure of very young clusters may hold important clues to the structure
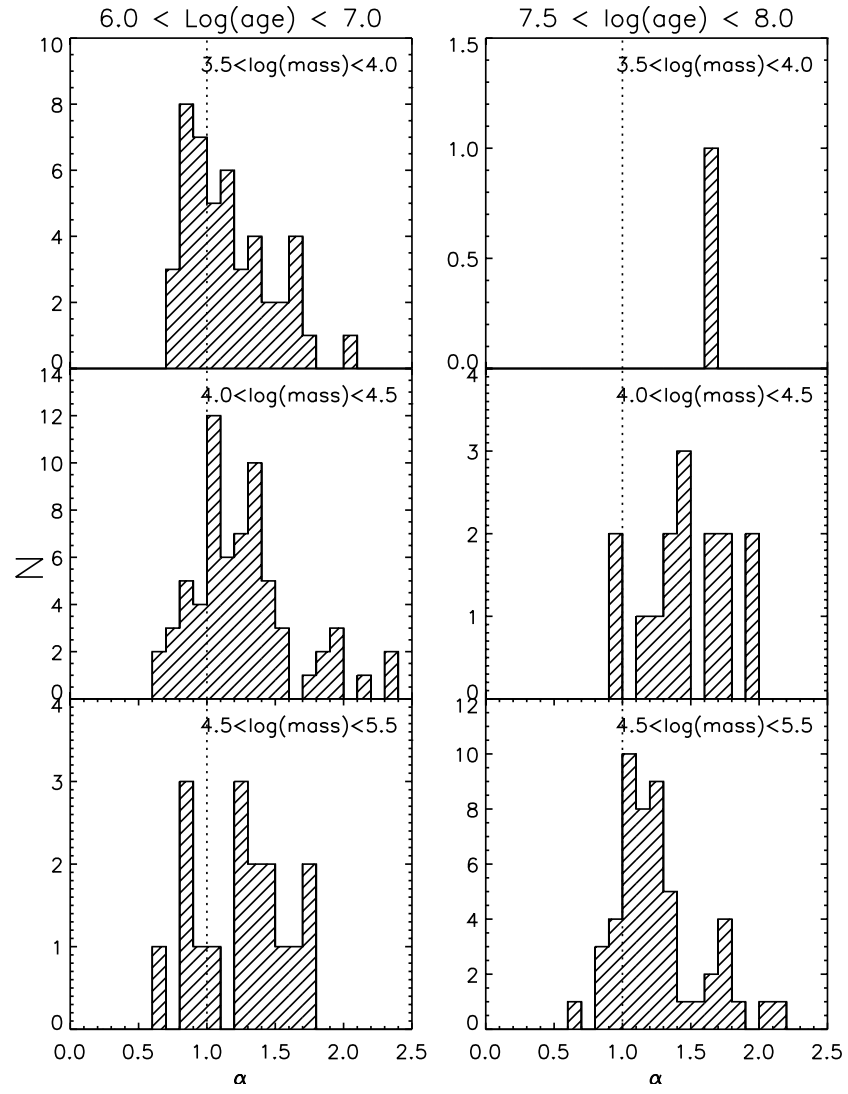

Fig. 13. Envelope slopes in different mass bins for two different ages.

of the progenitor clouds out of which the clusters formed, although it may prove challenging to disentangle this from the effects of early dynamical evolution.

\subsection{Cluster sizes versus age and mass}

Figures 14 and 15 show $F W H M$ and $R_{\text {eff }}$ versus age and mass for the combined cluster samples. Clusters older than $1 \mathrm{Gyr}$ are excluded from the plots because of large uncertainties on the ages (and therefore also on the masses derived from integrated photometry). The masses were estimated using $M / L$ ratios from the Bruzual \& Charlot models, assuming a Salpeter IMF from $0.1 M_{\odot}-100 M_{\odot}$. Absolute masses are sensitive to the shape and lower-mass cut-off of the IMF, and would be $\sim 30 \%$ lower for a Kroupa (2002) IMF, but this is not important for the relative comparison attempted here. In Fig. 15, clusters with $\alpha \leq 1$ and $\alpha>1$ are shown with different symbols (diamonds and plus markers). The dashed lines superimposed on the lower panels (size vs. mass) of each figure represent the size $\propto M^{1 / 3}$ relation corresponding to constant cluster density. Typical error bars are also shown in the top right corner in each of the lower plots.

Neither Fig. 14 nor Fig. 15 shows any strong evidence for a general correlation between cluster size and age. Since $F W H M$ is roughly equivalent to $2 \times r_{\mathrm{c}}$, it is interesting to note that Mackey \& Gilmore (2003) found a strong correlation between core radius and age for clusters in the LMC (confirming earlier results by Elson et al. 1989). For ages $<10^{8}$ years, there are essentially no clusters in the LMC with $r_{\mathrm{c}}>2.5 \mathrm{pc}$, while 

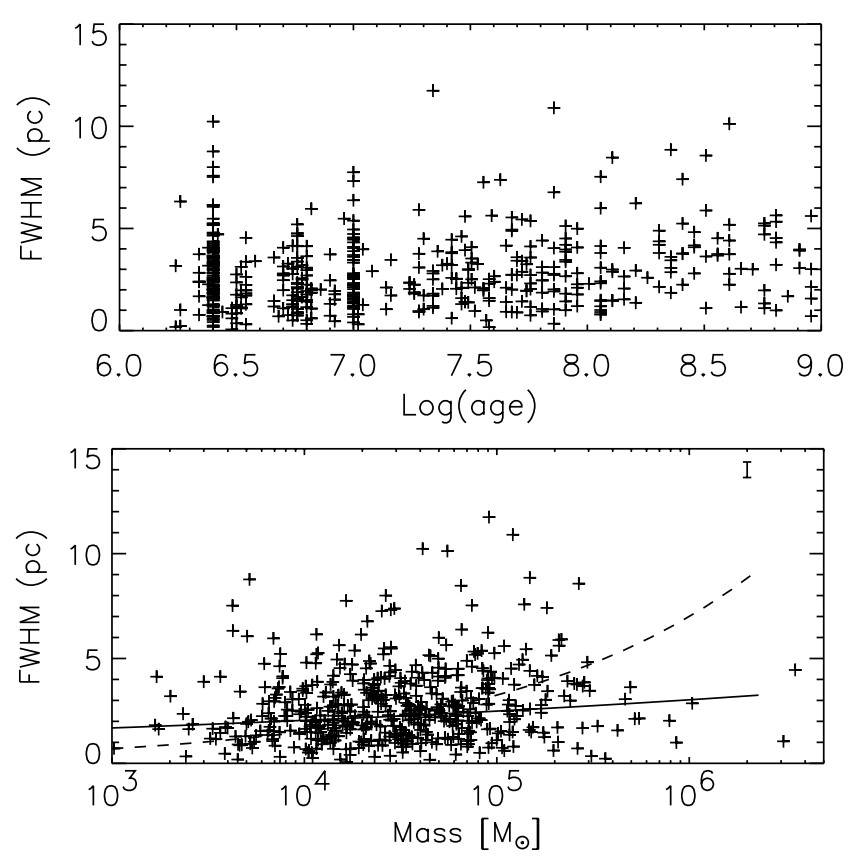

Fig. 14. Cluster FWHM versus age (top) and mass (bottom). The dashed curve in the lower panel represents a relation corresponding to $F W H M \propto M^{1 / 3}$ (constant density). The solid line is a least-squares power-law fit to $F W H M$ vs. mass.
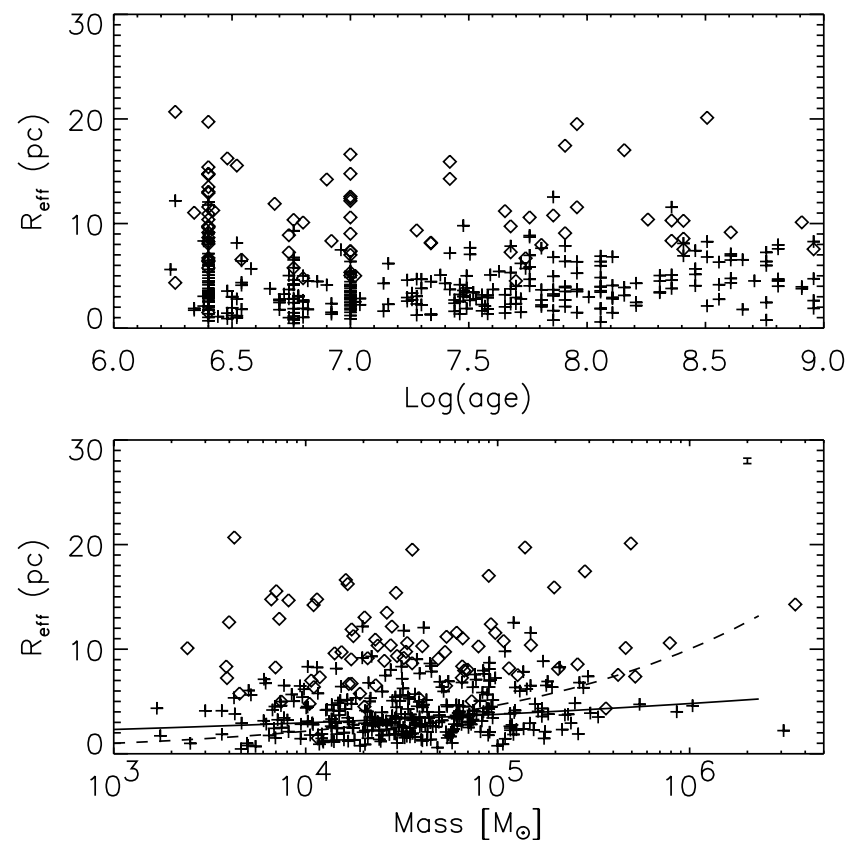

Fig. 15. Effective radius $\left(R_{\text {eff }}\right)$ versus age (top) and mass (bottom panel). Clusters with $\alpha \leq 1$ and $\alpha>1$ are shown with diamonds and plus markers, respectively. The dashed curve in the lower panel represents a relation corresponding $R_{\text {eff }} \propto M^{1 / 3}$ (constant density). The solid line is a least-squares power-law fit to $R_{\text {eff }}$ vs. mass for clusters with $\alpha>1$.

clusters with ages $\sim 10^{9}$ years show a full range of core radii from less than 1 to $8 \mathrm{pc}$. While it cannot be ruled out that the most extended clusters are missing from the sample, Fig. 14 shows no strong $r_{\mathrm{c}}$-age relation similar to that in the LMC.
Likewise, the size-mass plots show no strong correlations. The solid line in the bottom panel of Fig. 14 is a least-squares power-law fit of the form

$F W H M=A\left(M / M_{\odot}\right)^{B}$

where $M$ is the mass and $F W H M$ is measured in pc. The bestfitting parameter values are $A=0.94 \pm 0.25 \mathrm{pc}$ and $B=$ $0.08 \pm 0.03$. The fit formally indicates a $\sim 3 \sigma$ correlation between $F W H M$ and mass, but with a large scatter. The errors on the coefficients $A$ and $B$ are the formal errors from the leastsquares fit, but a jack-knife test yields nearly identical error estimates. The size cut may introduce a bias against more compact, low-mass objects, but the fit does not change substantially even if restricted to objects more massive than $10^{4} M_{\odot}$. While no strong constraints can be put on a $F W H M$ vs. mass relation based on Fig. 14, the formal relation derived from the leastsquares fit is shallower than the $M^{1 / 3}$ relation corresponding to constant core density (indicated by the dashed line). A similar fit is carried out for the $R_{\text {eff }}$ vs. $M$ data in Fig. 15:

$R_{\mathrm{eff}}=A\left(M / M_{\odot}\right)^{B}$,

but only for clusters with $1<\alpha<5$. Here, the best-fitting parameters are $A=1.12 \pm 0.35 \mathrm{pc}$ and $B=0.10 \pm 0.03$, quite similar to the slope of the $F W H M$ vs. mass relation in Fig. 14 and again only significant at about the $3 \sigma$ level. Formally, the slope is slightly steeper than (but compatible with) the $r \propto L^{0.07}$ relation found by Zepf et al. (1999) for young clusters in NGC 3256. As for the mean $R_{\text {eff }}$ values in Table 9, an important caveat is that the derived $R_{\text {eff }}$ are sensitive to the somewhat arbitrary truncation of the luminosity profiles at $50 \mathrm{pc}$. The slope of the $R_{\text {eff }}$ vs. $M$ relation remains stable at least for cut-offs between 30 and $100 \mathrm{pc}$, even if the clusters with $\alpha<1$ are included in the fit (but the normalisation changes somewhat). As in the fit to FWHM vs. mass (and Table 9), the individual datapoints are not weighted by their errors.

Using the HST photometry in $F 547 M, F 555 W$ or $F 606 W$ in Table 5 instead of the ground-based data to estimate the $V$-band luminosities of the clusters has no significant effect on the $F W H M$ vs. $M$ and $R_{\text {eff }}$ vs. $M$ relations. For $R_{\text {eff }}$ vs. $M$, the slope $B$ decreases by only 0.003 , while for $F W H M$ vs. $M$ it decreases by 0.014 , in both cases well within the formal uncertainties on the fits. Note, however, that the ages still have to be estimated from the ground-based photometry.

The high degree of crowding in Fig. 15 makes it difficult to visually assess to what extent the two curves agree with the data. A somewhat different representation of the same data is shown in Fig. 16, where the $R_{\text {eff }}$ distributions are shown for five separate mass bins. The sample with $\alpha>1$ is shown with shaded histograms, while the sample including clusters with $\alpha \leq 1$ is shown with outlined histograms. In each panel, the range of sizes corresponding to an $R_{\mathrm{eff}} \propto M^{1 / 3}$ scaling is also indicated (using the same arbitrary normalisation as in Fig. 15, not taking any scatter into account). The paucity of objects in the $0-1 \mathrm{pc}$ bin is due to the size cut, but even if one accounts for the fact that the most compact clusters may have been systematically excluded, it seems difficult to reconcile the overall $R_{\text {eff }}$ distributions in Fig. 16 with a constant-density relation. 


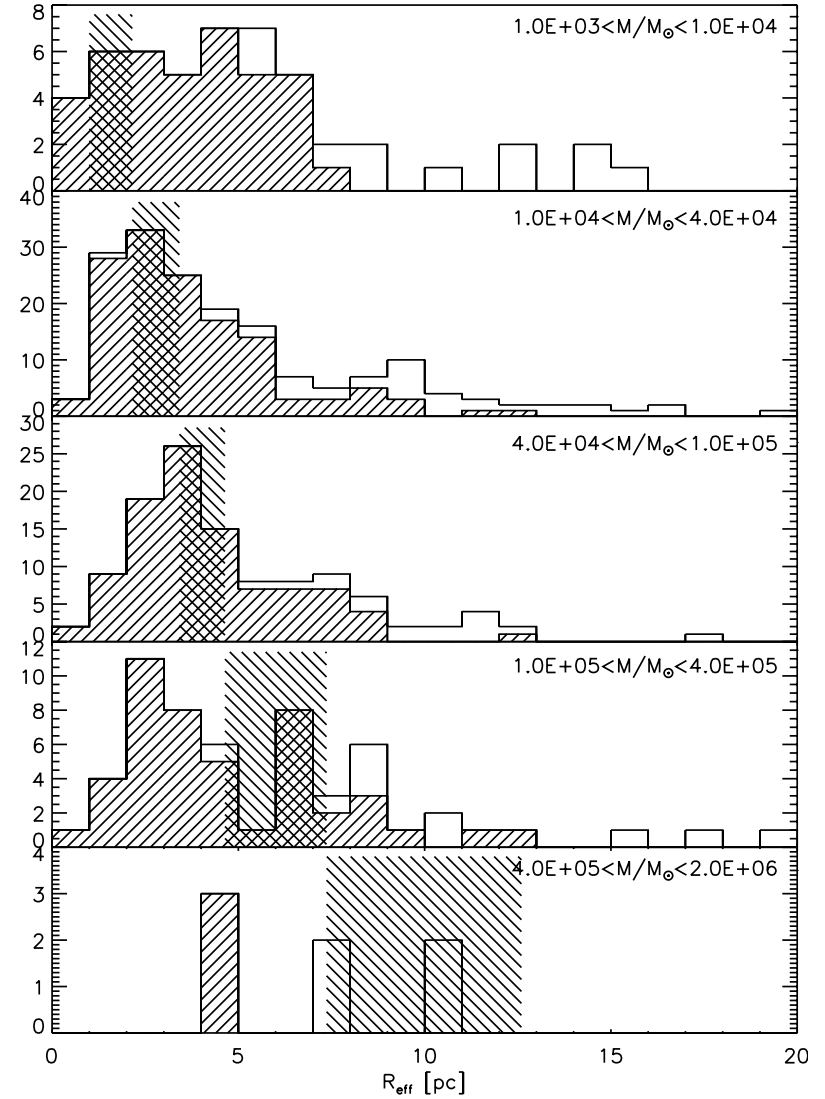

Fig. 16. Histograms of the $R_{\text {eff }}$ distributions in four mass ranges. Filled histograms are for clusters with $\alpha>1$, while the outlined histograms include clusters with $\alpha \leq 1$. The shaded areas indicate the range of sizes in each mass interval corresponding to an $R_{\mathrm{eff}} \propto M^{1 / 3}$ relation.

Although completeness effects are difficult to quantify, they would most likely tend to work against the detection of faint, extended clusters, thereby strengthening rather than weakening any existing size-mass trend. Thus, while the size-mass trends suggested by Figs. 14 and 15 remain quantitatively uncertain, the current data seem to imply that any size-mass trend, if it exists, is substantially shallower than for a constant-density relation.

\subsection{Environment}

While more subjective than the measurements of FWHM, $\alpha$ and $R_{\text {eff }}$, the comment flags in Table 3 hold useful information about the surroundings of each cluster. It is reiterated that the comment flags are based on visual inspection of a fairly heterogeneous dataset, and that the morphology of cluster candidates can be quite wavelength-dependent. Note, however, that the comment codes are based on visual inspection of all available images of each cluster, and the majority of the clusters have imaging in a $B-, V$ - or $I$-band equivalent filter. Table 10 lists the fraction of cluster candidates with comment flags a/c/d (all of which are likely indicators of multiplicity) and e/f/g/h (more general crowding indicators). These statistics are given both for the best cluster candidates with structural parameters determinations (i.e. Fit-OK = YES and Type 1), as well as for
Table 10. Statistics on comment flags in different age intervals.

\begin{tabular}{crrrrr}
\hline \hline Age range & \multicolumn{2}{c}{ Fit-OK = YES } & & \multicolumn{2}{c}{ All } \\
& Type $=1$ & & & \\
& a/c/d & e/f/g/h & Type $=2$ & a/c/d & e/f/g/h \\
\hline $6.0<\log ($ age $)<7.0$ & $36 \%$ & $2 \%$ & $42 \%$ & $54 \%$ & $19 \%$ \\
$7.0<\log ($ age $)<7.5$ & $22 \%$ & $0 \%$ & $22 \%$ & $32 \%$ & $4 \%$ \\
$7.5<\log ($ age $)<8.0$ & $16 \%$ & $0 \%$ & $25 \%$ & $29 \%$ & $7 \%$ \\
$8.0<\log ($ age $)<9.0$ & $10 \%$ & $0 \%$ & $7 \%$ & $14 \%$ & $0 \%$ \\
\hline
\end{tabular}

all potential cluster candidates including those without reliable fits (Fit-OK = YES/NO and Type 1/2). Data for all galaxies are included in Table 10, but the numbers remain unchanged within 1-2\% even if NGC 2997, NGC 7793 and NGC 7424 are omitted as in the previous sections.

Very few clusters with Fit-OK = YES have any of the /e/f/g/h flags set. This is no coincidence, because these flags indicate exactly those conditions which would make profile fits uncertain. The table indicates a strong evolution in the environment as a function of age. Most of the cluster candidates in crowded environments (flags e/f/g/h) are younger than $10^{7}$ years. The tendency for the crowding to decrease with age is probably a consequence of fading and dispersion of the surrounding stellar population. Assuming typical velocity dispersions of a few $\mathrm{km} \mathrm{s}^{-1}$ within (unbound) star forming regions, the expansion will amount to a few tens of $\mathrm{pc}$ in $10^{7}$ years.

Of the objects in the youngest age bin, $42 \%$ were classified as "uncertain" (type 2). This underscores the fundamental difficulty of identifying the youngest clusters. The problem of defining an appropriate selection criterion for bona-fide clusters is far from trivial. Sometimes the main problem is simply that an object is only barely resolved. In such cases, better angular resolution would help confirm or rule out the cluster nature. For objects with complex morphology it can be difficult to determine whether or not an object is a true star cluster, even if resolution would otherwise not be a problem. Examples can be seen in Fig. 3 and in panels (e), (f) and (g) of Fig. 2. In these cases, it is difficult to determine whether a well-defined stellar cluster is present. For low-mass clusters of low age, an additional problem is that the integrated light can be dominated by a few luminous stars, making it difficult to distinguish such objects from random superpositions of individual stars along the line-of-sight.

As pointed out in the introduction, most stars probably form in clusters, but only a small fraction of young embedded clusters survive as bound objects. It may also happen that only a fraction of the stars in a cluster remain bound, while the rest disperse away (Kroupa 2001). Thus, a few Myr-old concentration of stars may be a bound star cluster, a bound core surrounded by an expanding envelope, or an entirely unbound association which will soon disperse away completely. Other star formation may also be taking place nearby, perhaps triggered by the young cluster. So it is not surprising that a large fraction of the youngest objects have a messy morphology.

The age distribution of double or multiple objects does not appear to be as strongly peaked at young ages, with some objects flagged " $\mathrm{a} / \mathrm{c} / \mathrm{d}$ " even in the oldest age bin. It is possible 
that at least a fraction of these objects are genuine double clusters, similar to those found in the Large Magellanic Cloud. The LMC binary clusters tend to be predominantly young, though a few pairs as old as several times $10^{8}$ years exist (Dieball et al. 2002). This seems to be consistent with the decreasing fraction of multiple objects at high ages in Table 10. However, because the main source of photometry in this paper is ground-based imaging, no information is available about possible colour/age differences between the components in such pairs. Multi-colour HST imaging, especially including $U$-band data, would allow a more thorough investigation of double clusters and make a comparison with the LMC sample possible.

\section{Summary and conclusions}

Using a combination of HST/WFPC2 imaging and groundbased $U B V I$ photometry, a catalogue of cluster candidates in 18 nearby spiral galaxies has been compiled. Only objects with a $S / N>50$ on the HST images (within an $r=5$ pixels aperture) were included, allowing for a detailed analysis of the structure of individual clusters. Analytic profile fits of the form $P(r) \propto\left(1+\left(r / r_{\mathrm{c}}\right)^{2}\right)^{-\alpha}$ were carried out, including a proper modelling of the HST/WFPC2 PSF, and allowing both the core radius $r_{\mathrm{c}}$ and envelope slope parameter $\alpha$ to vary. Each cluster candidate has also been visually inspected and comment flags relating to crowding and multiplicity are given. These comment flags, combined with the photometric data and structural parameters, may be helpful when using the list of cluster candidates to select targets e.g. for spectroscopic studies.

The HST imaging indicates a mean contamination rate of $20 \%$ or less for the ground-based cluster surveys in Papers IIII, although the contamination rates in some individual galaxies (most notably NGC 5204) are higher. However, the relation between specific $U$-band luminosity of the cluster systems $T_{L}(U)$ and $\Sigma_{\mathrm{SFR}}$ (Paper III) remains valid after correction for contamination. Because very few clusters remain after the contamination correction in some galaxies, sampling the cluster populations to fainter magnitudes than the limits defined in Papers I-III would probably reduce the scatter in the $T_{L}(U)$ vs. $\Sigma_{\text {SFR }}$ relation.

The cluster catalogue has been used to investigate trends and relations between various cluster properties, although the analysis is complicated by the fact that selection effects are difficult or impossible to control with a dataset as heterogeneous as the one used here. Most clusters have FWHM less than about $8 \mathrm{pc}$ with a formal mean of about $2.7 \mathrm{pc}$, corresponding to a mean core radius $\left\langle r_{\mathrm{c}}\right\rangle \approx 1.3 \mathrm{pc}$, but very compact clusters may be missing because of the size cut imposed in order to exclude point sources (individual stars). For the subset of the clusters which have $1<\alpha<5$ and therefore reasonably reliable measurements of the effective radius $R_{\text {eff }}$, the mean value is $\left\langle R_{\text {eff }}\right\rangle=3.94 \pm 0.12 \mathrm{pc}$, but this mean value may again be affected by a selection bias against the most compact clusters. The effective radii are also sensitive to the poorly constrained behaviour of the luminosity profiles at large radii. Here, the profiles are truncated at $50 \mathrm{pc}$. The structural parameters show little or no variation from galaxy to galaxy, especially when considering that the distances are not always known very accurately. In particular, the effective radii are uncorrelated with the host galaxy area-normalised star formation rate, and are also very similar to those of open and globular clusters in the Milky Way, globular clusters in early-type galaxies, and young clusters in merger galaxies and starbursts. It is quite remarkable that the sizes of stellar clusters are largely invariant with respect to the properties of the parent galaxy. Physical parameters such as gas density and -pressure probably play a major role in regulating the star formation rate (Kennicutt 1998), but while these factors may affect the formation efficiency of bound clusters (Paper III) they do not appear to have a strong impact on the structure of the clusters themselves, once formed. Exceptions to this rule do exist, including the "faint fuzzy" star clusters observed in some lenticular galaxies (Brodie \& Larsen 2002), and there is a general trend for the sizes of globular clusters to increase as a function of galactocentric distance (van den Bergh et al. 1991)

While both the $F W H M$ and $R_{\text {eff }}$ are found to correlate with cluster mass, least-squares power-law fits yield slopes that are substantially shallower than for a constant-density relation, implying an increase in cluster density as a function of mass. Qualitatively, these results are in agreement with previous data for young star clusters as well as old globular clusters. Quantitatively, the relations show a large scatter and remain uncertain. Ashman \& Zepf (2001) have argued that an increasing star formation efficiency as a function of cluster mass may explain (at least partially) the lack of a strong size-mass relation.

Many of the youngest clusters have extended, shallow outer envelopes. This tendency seems to be a general one, noted previously for a few isolated cases in the Antennae, NGC 6946, and for LMC clusters. The structure of these young objects may hold important clues to the early dynamical evolution of clusters and the density distribution of the parent proto-cluster clouds. Older clusters gradually evolve towards King-type profiles with a finite tidal radius.

Finally, a strong correlation between cluster age and crowding is found, with most of the strongly crowded clusters having ages $<10^{7}$ years. About $1 / 3-1 / 2$ of these young objects are double or multiple sources, but the identification as bonafide clusters is often uncertain even on WFPC2 images in these fairly nearby galaxies. Future multi-colour imaging with the Advanced Camera for Surveys on HST should help resolve many of the difficulties encountered in this study.

Acknowledgements. This work was partially supported by National Science Foundation grant AST 02-06139 and by HST grant AR09523. I am grateful to T. Richtler and S. M. Fall for several helpful comments, and to the referee, R. de Grijs, for a very detailed report which helped improve the paper.

\section{References}

Anders, P., \& Fritze-v. Alvensleben, U. 2003, A\&A, 401, 1063 Ashman, K. M., \& Zepf, S. E. 2001, AJ, 122, 1888

Billett, O. H., Hunter, D. A., \& Elmegreen, B. G. 2002, AJ, 123, 1454

Brodie, J. P., \& Larsen, S. S. 2002, AJ, 124, 1393

Burstein, D., \& Heiles, C. 1984, ApJS, 54, 33

Cardelli, J. A., Clayton, G. C., \& Mathis, J. S. 1989, ApJ, 345, 245

Carpenter, J. M. 2000, AJ, 120, 3139 
Chandar, R., Bianchi, L., Ford, H. C., \& Salasnich, B. 1999, PASP, 111,794

Christian, C. A., \& Schommer, R. A. 1982, ApJS, 49, 405

Christian, C. A., \& Schommer, R. A. 1988, AJ, 95, 704

de Grijs, R., Lee, J. T., Clemencia Mora Herrera, M, Fritze-v. Alvensleben, U., \& Anders, P. 2003, New Astron., 8,155

Dieball, A., Müller, H., \& Grebel, E. K. 2002, A\&A, 391, 547

Elmegreen, B. G. 1983, MNRAS, 203, 1011

Elson, R. A. W., \& Fall, S. M. 1985, ApJ, 299, 211

Elson, R. A. W., Fall, S. M., \& Freeman, K. C. 1987, ApJ, 323, 54

Elson, R. A. W., Freeman, K. C., \& Lauer, T. R. 1989, ApJ, 347, L69

Girardi, L., Chiosi, C., Bertelli, G., \& Bressan, A. 1995, A\&A, 298, 87

Girardi, L., 2000 in Massive Stellar Clusters, ed. A. Lançon, \& C. Boily, ASP Conf. Ser., 211

Harris, W. E. 1996, AJ, 112, 1487

Hodge, P. W. 1961, ApJ, 133, 413

Holtzman, J. A., Burrows, C. J., Casertano, S., et al. 1995, PASP, 107, 1065

Janes, K. A., Tilley, C., \& Lyngå, G. 1988, AJ, 95, 771

Kennicutt, R. C. 1998, ARA\&A, 36, 189

King, I. R. 1962, AJ, 67, 471

King, I. R. 1966, AJ, 71, 64

Krist, J., \& Hook, R. 1997, The Tiny Tim User's Guide, STScI

Kroupa, P., Aarseth, S., \& Hurley, J. 2001, MNRAS, 321, 699

Kroupa, P. 2002, Science, 295, 82

Kundu, A., \& Whitmore, B. C. 2001, AJ, 121, 2950
Lada, C. J., \& Lada, E. A. 2003, ARA\&A, in press [astro-ph/0301540]

Larsen, S. S. 1999, A\&AS, 139, 393 (Paper II)

Larsen, S. S., \& Richtler, T. 1999, A\&A, 345, 59 (Paper I)

Larsen, S. S., \& Brodie, J. P., 2000, AJ, 120, 2938

Larsen, S. S., Brodie, J. P., Elmegreen, B. G., et al. 2001, ApJ, 556, 801

Larsen, S. S., \& Richtler, T. 2000, A\&A, 354, 836 (Paper III)

Larsen, S. S. 2002, AJ, 124, 1393

Mackey, A. D., \& Gilmore, G. F. 2003, MNRAS, 338, 85

Meurer, G. R., Heckman, T. M., Leitherer, C., et al. 1995, AJ, 110, 2665

Monet, D., Bird A., Canzian, B., et al. 1998, The USNO-A2.0 Catalogue (Washington DC: US Naval Observatory)

Richtler, T. 1993, in The Globular Cluster-Galaxy Connection, ed. G. H. Smith, \& J. P. Brodie, ASP, 375

Schlegel, D. J., Finkbeiner, D. P., \& Davis, M. 1998, ApJ, 500, 525

Shapley, H., \& Nail, V. McKibben, 1951 AJ, 55, 249

Spitzer, L. 1987, Dynamical Evolution of Globular Clusters, Princeton Series in Astrophysics (Princeton University Press)

Stetson, P. 1987, PASP, 99, 191

van den Bergh, S., Morbey, C., \& Pazder, J. 1991, ApJ, 375, 594

Whitmore, B. C. 2003, in STScI Symp. Ser. 14, ed. M. Livio [astro-ph/0012546]

Whitmore, B. C., Zhang, Q., Leitherer, C., et al. 1999, AJ, 118, 1551

Zepf, S. E., Ashman, K. M., English, J., Freeman, K. C., \& Sharples, R. M. 1999, AJ, 118, 752 